\title{
Drosophila USP22/non-stop regulates the Hippo pathway to polarise the actin cytoskeleton during collective border cell migration
}

\author{
Hammed Badmos ${ }^{1,2}$, Neville Cobbe ${ }^{1}$, Amy Campbell ${ }^{1}$, Daimark Bennett ${ }^{1,2}$ \\ 1 Department of Biochemistry, Institute of Integrative Biology, University of Liverpool, Crown Street, Liverpool, L69 7ZB, UK \\ 2 Department of Molecular Physiology \& Cell Signalling, Institute of Systems, Molecular and Integrative Biology, University of Liver- \\ pool, Crown Street, Liverpool, L69 7ZB, UK
}

Polarisation of the actin cytoskeleton is vital for the collective migration of cells in vivo. During invasive border cell migration in Drosophila, actin polarisation is directly controlled by Hippo pathway components, which reside at contacts between border cells in the cluster. Here we identify, in a genetic screen for deubiquitinating enzymes involved in border cell mi- 200 gration, an essential role for non-stop/USP22 in the expression of Hippo pathway components expanded and merlin; loss of non-stop function consequently leads to a redistribution of F-actin and the polarity determinant Crumbs, loss of polarised actin protrusions and premature tumbling of the border cell cluster. Non-stop is a component of the Spt-Ada-Gen5acetyltransferase (SAGA) transcriptional coactivator complex, but SAGA's histone acetyltransferase module, which does not bind to expanded or merlin, is dispensable for migration. Taken together, our results uncover novel roles for SAGA-independent nonstop/USP22 in Hippo-mediated collective cell migration, which may help guide studies in other systems where USP22 is necessary for cell motility and invasion.

\section{Introduction}

Tightly regulated cell migration is vital for normal development and aberrant migration is involved in a number of human diseases, including tumour invasion and cancer metastasis, inflammatory diseases, and various birth abnormalities (Schumacher, 2019; Stuelten et al., 2018). In many instances, cells move by the process of collective migration in vivo, whereby migratory cells remain connected by cell-cell junctions, show group polarisation and coordinated cytoskeletal dynamics (Haeger et al., 2015; Mishra et al., 2019; Norden and Lecaudey, 2019). This mode of migration is exemplified by the movement of border cells in Drosophila (video S1). In this process, a cluster of five to eight cells are recruited from the follicular epithelium in the ovary by a pair of non-motile polar cells. Both cell types migrate as a cluster from the anterior basal lamina of the egg chamber, invading the underlying germ line, to the anterior border of the oocyte where they are involved in patterning prior to egg fertilization (Montell et al., 2012).

Studies of this process over the past 20 years have iden- tified key features of the genetic programme required for border cell migration, which control the specification of the migratory cluster (Bai et al., 2000; Montell et al., 1992; Silver and Montell, 2001), organisation of cluster polarity and detachment from the epithelium (Abdelilah-Seyfried et al., 2003; McDonald et al., 2008; Pinheiro and Montell, 2004), timing of migration (Godt and Tepass, 2009; Jang et al., 2009), adhesion of the cluster (Cai et al., 2014; Niewiadomska et al., 1999) and guidance to the oocyte (Bianco et al., 2007; Duchek and Rorth, 2001; Duchek et al., 2001; McDonald et al., 2003). Details have also emerged regarding the dynamic organisation of the actin cytoskeleton which is an essential driver of this process (Plutoni et al., 2019), with recent studies identifying an important role for the Hippo pathway in linking determinants of cell polarity with polarisation of the actin cytoskeleton in migrating clusters (Lucas et al., 2013). Our understanding of the interplay between polarity determinants and the actin cytoskeleton however remain incomplete, as does knowledge of the regulatory networks responsible for first establishing this polarity.

Ubiquitination of proteins by ubiquitin E3 ligases and removal by deubiquitinating enzymes (DUBs) plays important roles in regulating a raft of intracellular functions from protein stability and enzyme activity to receptor internalization and protein-protein interactions (Clague et al., 2013; Swatek and Komander, 2016). There is a growing body of evidence that ubiquitination plays roles in regulating the motility of single cells in culture (Cai et al., 2018), but little is known about its contribution to collective migration in vivo. Here we report our identification of nonstop (not) from a screen of DUBs involved in border cell migration. not encodes the USP22 orthologue in Drosophila (Martin et al., 1995), and is best known as the enzymatic component of the histone H2B DUB module of the SAGA transcriptional coactivator complex (Koutelou et al., 2010; Lee et al., 2011; Zhang et al., 2008). Histone modifications such as acetylation and ubiquitination are known to modulate the accessibility of genomic loci to transcriptional machinery, with ubiquitination being associated with both activation and repression (Weake and Workman, 2008). Correspondingly, SAGA is associated with the enhancers, promoters and sites of paused RNA polymerase II at genes in multiple tissues during Drosophila embryogenesis, and the Non-stop activity within SAGA is required for full expression of tissue-specific 
genes (Weake et al., 2011).

Previous work has revealed essential roles for non-stop/ USP22 during embryogenesis in Drosophila and mammals (Li et al., 2017; Lin et al., 2012), as well as in neural development (Weake et al., 2008) and lineage specification (Kosinsky et al., 2015). In the Drosophila nervous system, loss-of-function mutations in non-stop are associated with defects in the migration of a subset of glial cells to their appropriate position in the developing optic lobe and subsequent targeting of photoreceptor axons in the lamina (Martin et al., 1995; Poeck et al., 2001). The underlying mechanisms are not fully understood, but it has recently been suggested that this role may be mediated in part by a SAGA- independent role of Not in deubiquitinating and stabilising the actin regulator Scar (Cloud et al., 2019). Here we find that, in collective border cell migration, not functions independently of both Scar and SAGA to regulate the expression of two upstream components of the hippo pathway, resulting in the loss of F-actin polarity, the mislocalisation of polarity determinants, a change in the size and orientation of cellular protrusions and the loss of polarised migration, placing non-stop at the top of a regulatory network underlying collective migration.

\section{Results}

non-stop is required for invasive border cell migration. We identified the Drosophila USP22 homologue non -stop in an RNA interference (RNAi) screen for deubiquitinases (DUBs) required for border cell migration. Wildtype border cell clusters normally reach the oocyte by stage 10 of oogenesis, whereas expression of transgenic inverted repeat constructs for non-stop $\left(n o t^{t R}\right)$ in the outer border cells severely delayed border cell migration (mean percentage migration of the distance to the oocyte \pm SEM was $2.5 \pm 2.5 \%, \mathrm{n}=40$, Student's t-test, $P<0.0001$ ) (Fig. $1 \mathrm{~A}$ -E). These migration defects could be significantly rescued by a full-length synthetic RNAi-resistant transgene (not ${ }^{+r}$, see Methods) confirming the requirement for nonstop in migration (Fig.1D,E). Incomplete rescue is most likely an indication of some off-target effects of not $t^{R}$.

Expression of not $^{+r}$ alone in both polar and outer border
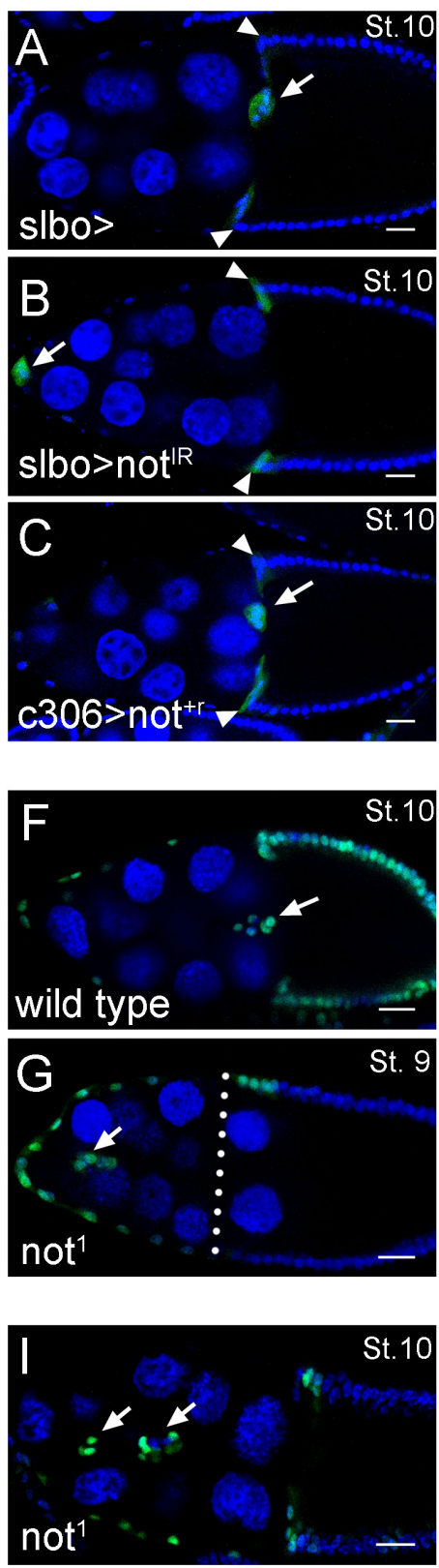
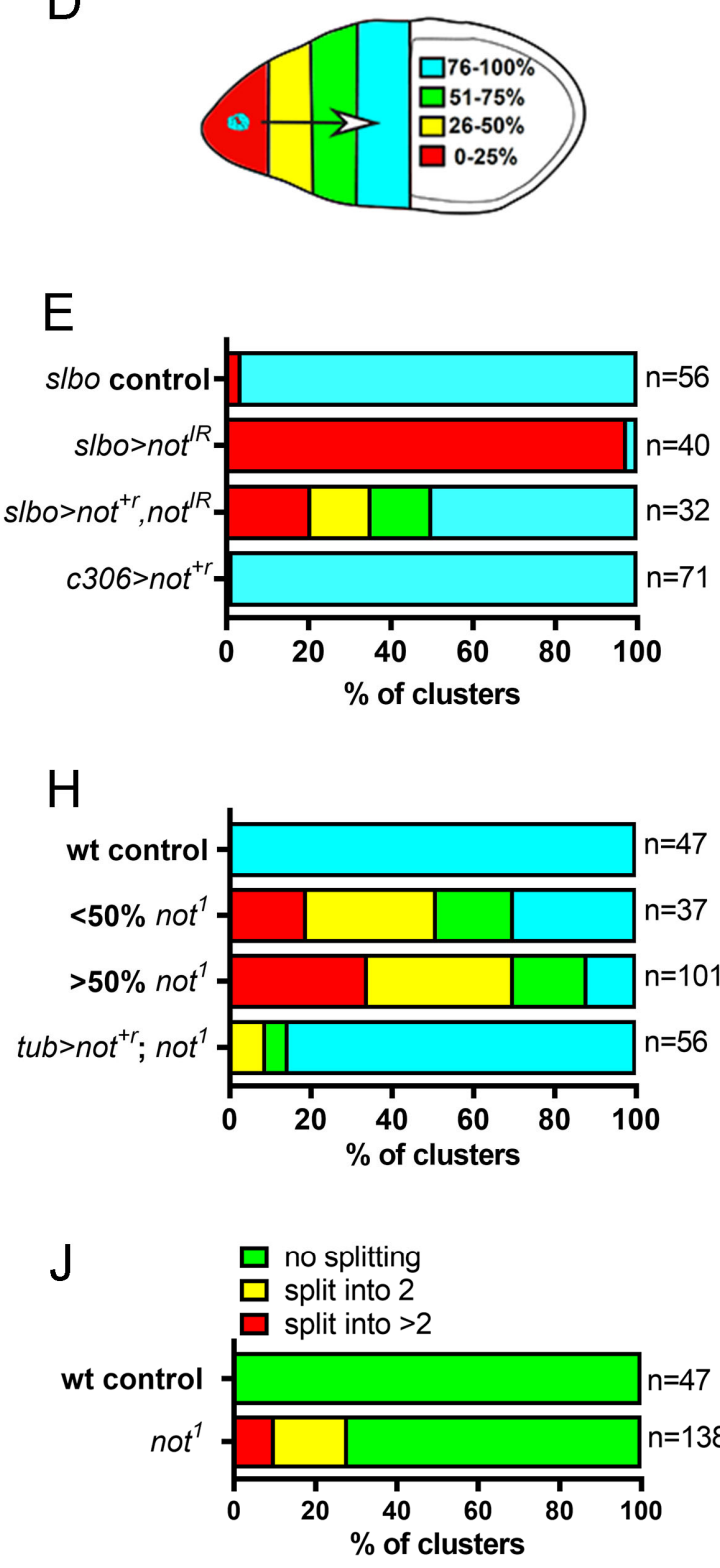

Fig.1 non-stop is required for invasive border cell migration. A-D, Confocal micrographs of egg chambers at stage 10 labelled with GFP (green) under the control of slbo-GAL4 to mark border cells (arrows) and TOPRO-3 (blue) to stain all nuclei. Anterior is left, posterior is right. Some GFP expression is also evident in centripetal follicle cells (arrowhead). Bars, $25 \mu \mathrm{m}$. A, Image of slbo-GAL4 control (slbo>) showing complete migration of the border cell cluster. B, RNAi knockdown of not under the control of slboGAL4 (slbo>not ${ }^{\mathrm{R}}$ ) abrogates border cell migra-

tion. C, In contrast, overexpression of $n o t^{+r}$ in the whole border cell cluster using c306-GAL4 $\left(c 306>\right.$ not $^{+r}$ ) did not affect migration, indicating non-stop is not limiting for migration. Clusters expressing not $^{+r}$ with SIbo-GAL4 also migrated normally (not shown). D, Migration index for quantification of border cell migration at stage 10, see Methods. E, Stacked bar chart summarising migration defects in the indicated genotypes $(n=$ number of egg chambers). The effect of not RNAi knockdown can be partially rescued by transgenic overexpression of RNAi-resistant not $\left(\mathrm{slbo}>\right.$ not $^{+r}$ not $\left.^{\prime R}\right)$. F-G, Confocal micrographs of egg chambers labelled with GFP (green) to mark clones of cells induced with the MARCM technique and TOPRO-3 (blue) to stain all nuclei. Bars, $25 \mu \mathrm{m}$. Compared to control clones, which routinely complete migration at stage $10(\mathbf{F})$, not $^{1}$ mutant border cell clusters display defective migration, with clusters lagging behind overlying centripetal cells (position marked with dotted line) at stage 9 (G). H, Quantitation of migration defects at stage 10 , reveal that the clusters containing $>50 \%$ mutant cells are more severity affected than those with $<50 \%$ mutant cells in the cluster; migration is largely restored by not $^{+r}$ overexpression (tub>not ${ }^{+r} ;$ not $^{1}$ ). $n=$ number of egg chambers. I, Stage 10 egg chamber showing splitting of $n o t^{1}$ mutant border cell clusters; $18 \%$ of clusters displayed splitting into two groups of cells, $10 \%$ of clusters split into $>2$ groups of cells $(\mathbf{J}$, frequency of cluster splitting, $n=$ number of egg chambers). 
cells had no effect on migration (Fig.1C,E). To further confirm the requirement for non-stop in border cell migration, we generated homozygous clones for an amorphic non-stop mutant allele $\left(n o t^{1}\right)$. Notably, border cell clusters genetically mosaic for not $^{1}$ showed greatly retarded migration, with the severity of the effect being dependent on the proportion of mutant cells in the cluster (Fig.1F-H). Mean migration was reduced by $61.3 \pm 2.9 \% \quad(P<.0001$, $\mathrm{n}=101$ ) in clusters containing $>50 \%$ non-stop mutant cells compared to clusters with control clones, where cells migrated normally; these defects were almost fully rescued by transgenic expression of not $^{+r}($ Fig.1H). Unlike in controls, splitting of border cell clusters was also observed in $28 \%$ of stage 9 or 10 non-stop mutant egg chambers $(n=138)$ (Fig.1I-J), indicative of a defect in maintaining the integrity of border cell-border cell contact. Taken together, these data identify non-stop as a novel regulator of border cell migration. non-stop regulates polar cell number. At stage 8 of repress polar cell fate in these cells (Bai and Montell, oogenesis, a pair of anterior polar cells secrete Unpaired (Upd) ligand, which activates the JAK-STAT (Janus kinase-signal transducer and activation of transcription) signalling pathway in surrounding follicle cells, leading to the recruitment of 5-8 follicle cells into a migratory cluster (Beccari et al., 2002; Silver and Montell, 2001). To explore the requirement for non-stop in border cell signalling we looked at the expression of slbo, a downstream target of Upd-JAK/STAT signalling in the migratory outer border cells, which induces the expression of genes required for migration. The level of a transcriptional reporter, slbo-lacZ, was not significantly different between $n o t^{1}$ mutant cells and their wild type siblings within mosaic border cell clusters (Fig.2A-C; arbitrary units, mean intensity \pm SEM was $47 \pm 5.3$ for $n t^{1} n=21$, compared to 56 \pm 5.5 for controls $n=23, P=0.22$ ). non-stop was also not required for the expression pattern of Eyes absent (Fig.2D,E), which is expressed in outer border cells to
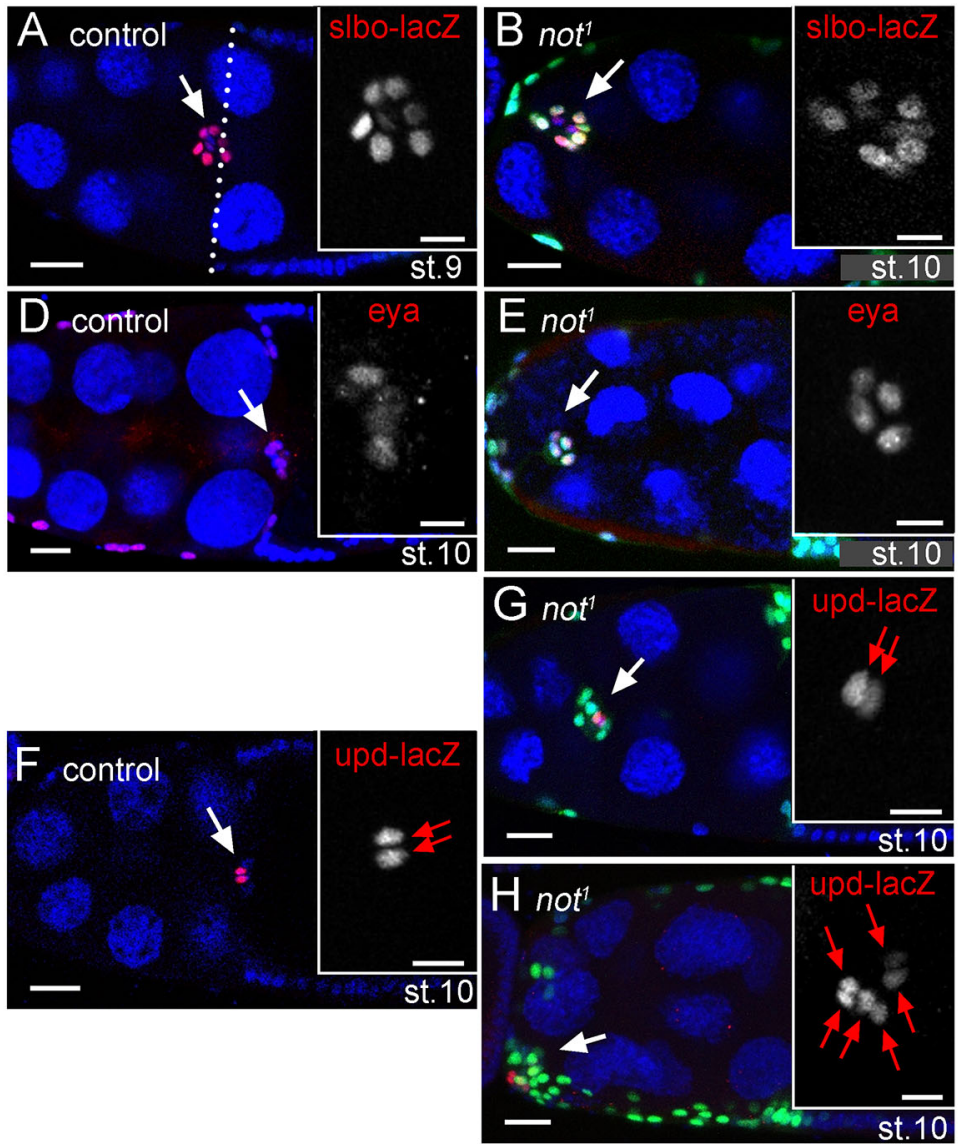
2002). Therefore, we conclude that non-stop does not non-stop is required for normal actin polarity in miaffect the expression levels of genes in migratory outer gratory border cells. Following their specification, borborder cells that specify their fate. When we looked at der cells undergo two phases of cell migration, an initial upstream signalling using a upd-lacZ reporter (Fig.2F-H), polarised phase, and a second phase that utilises collecwe observed that $32 \%$ of not $^{1}$ mutant clusters possessed tive migration (Bianco et al., 2007). In the initial phase, more than two upd-lacZ positive polar cells (Fig.2I; mean leader cells exhibit long, highly polarised F-actin protru\pm SEM was $2.47 \pm 0.12, n=53$ ), suggesting that some not ${ }^{1}$ sions that are required for adhesion to and migration polar cells continue proliferating after stage 2 of egg through the substratum (Fulga and Rorth, 2002). Later, Fchamber development when divisions would normally actin accumulates around the cortex of the cluster, as cease (Margolis and Spradling, 1995). not ${ }^{1}$ clusters also cells alternate their position in the cluster as they move contained on average a 1.7-fold higher number of border collectively (Bianco et al., 2007). In not ${ }^{1}$ mutant clones, cells than controls (Fig.2l; mean \pm SEM was $11.1 \pm 0.2$ for we observed a loss of initial F-actin polarity, and F-actin not $^{1} \mathrm{n}=138$, compared to $6.4 \pm 0.12$ for controls $n=47$, accumulation was subsequently not restrained to the $P<0.0001)$ and this was correlated with the number of cluster cortex but it also accumulated along border cellupd-lacZ positive polar cells (Fig.2J; multiple regression border cell junctions (Fig. 3A,B). Quantification of F-actin $\mathrm{R}^{2}=0.54, \mathrm{n}=53, P<0.0001$ ), suggesting the presence of staining confirmed a 2.6-fold shift in relative distribution additional polar cells led to the recruitment of additional towards the interior border cell junctions in not ${ }^{1}$ mutant border cells into the cluster. Clusters with more than two clusters compared to controls (two-way Anova P<0.0001, polar cells had a significantly reduced degree of migration Fig.3C). This change in distribution was rescued by transcompared to those with just two polar cells, (mean migra- genic not ${ }^{+r}$ overexpression (Fig.3C). When we examined tion was $3.8 \%(n=17)$ compared to $22.2 \%(n=36)$, respec- egg chambers by live imaging, we found that progressive tively, t-test $P=0.003$ ), suggesting that larger not $^{1}$ clusters migration was reduced by $80 \%$, from $0.45 \mu \mathrm{m} / \mathrm{min}$ in conhad particular difficulty in making their way successfully trols to $0.09 \mu \mathrm{m} / \mathrm{min}$ in not $^{1}$ mutant border cell clusters to the oocyte.

$(\mathrm{P}<0.01)$. This was accompanied by loss of initial F-actin polarity (Fig.4A,B, video S2 and S3) and a premature
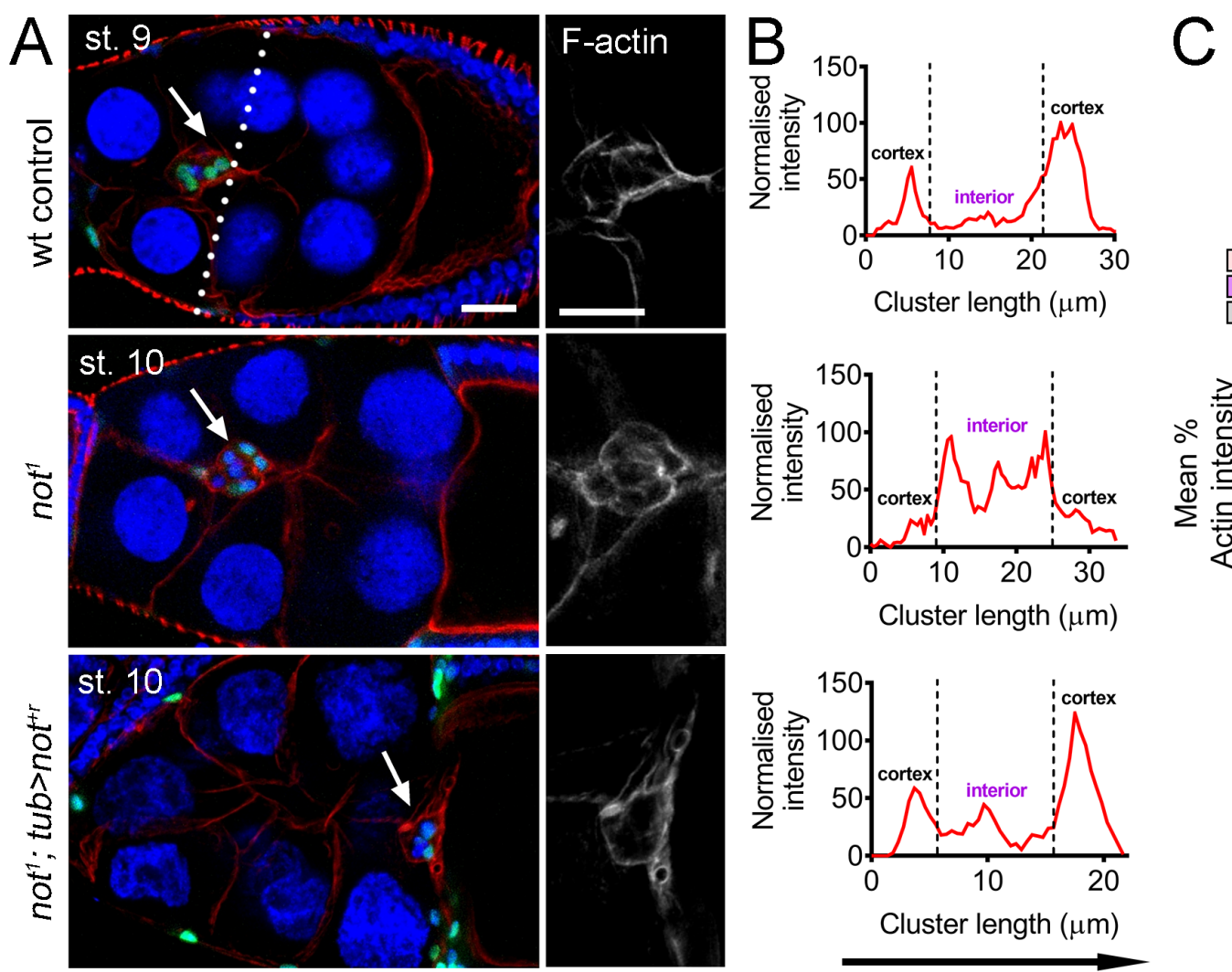

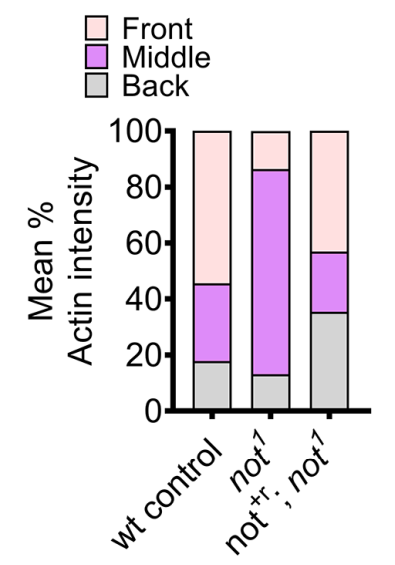

Fig.3 non-stop is required for normal actin polarity in migratory border cells. A, Confocal micrographs of egg chambers harbouring wild type, not $^{1}$ or rescued not $^{1}$ GFP-labelled clones ( not $^{1}$; tub>not ${ }^{+r}$ ) labelled with Phalloidin to visualise F-actin (red), TO-PRO-3 to label nuclei (blue). Egg chambers are stage 10 except the wt control, which is shown at mid-migration at stage 9 (dotted line indicates expected position of the cluster at this stage of migration). Border cell clusters are indicated with arrows. In wt, F-actin is normally polarised, with high levels around the cortex of the cluster, at border cell-nurse cell junctions. In contrast, in not ${ }^{1}$ clusters, F-actin predominantly accumulates at internal border cell-border cell junctions; this is rescued by transgenic overexpression of not ${ }^{+r}$. Bars are $25 \mu \mathrm{m}$ (RGB images) and $10 \mu \mathrm{m}$ for magnified grayscale images of $\mathrm{F}$ actin. B, Representative line scans of the same genotypes showing signal intensities of F-actin from anterior (left) to posterior (right), showing the change in F-actin profile in not ${ }^{1}$ mutant clusters. C, Mean ratios of area under curve for front, middle and back of the cluster derived from lines

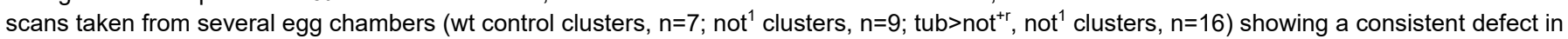
F-actin polarisation in not $^{1}$ clusters. 

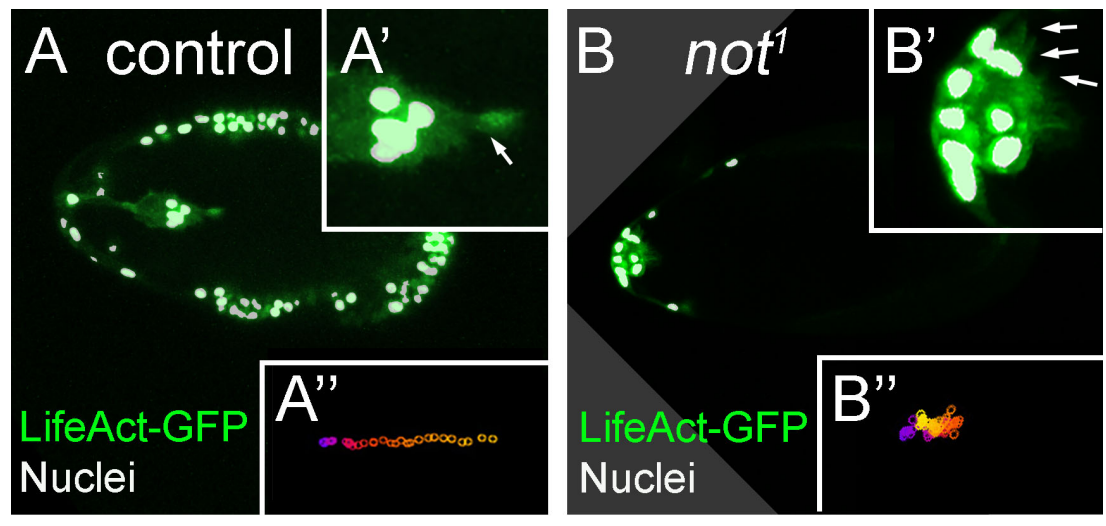

Fig.4 Loss of non-stop results in loss of polarised protrusions, retarded migration and early tumbling

A-B, Still images from time-lapse imaging of LifeAct-GFP -labelled border cells near the start of border cell migration with nuclear GFP-labelled MARCM clones labelled in white and LifeAct-GFP in green. $\mathbf{A}$ (inset $\mathbf{A}^{\prime}$, magnified image), Control egg chamber with clearly visible polarised F-actin protrusion at the leading edge of the cluster (arrow in $\mathbf{A}^{\prime}$ ), leading to progressive migration from anterior to posterior (track A", generated using a custom macro, (Poukkula et al., 2011)). In contrast, not ${ }^{1}$ clusters (B) display multiple shorter protrusions at different positions around the cluster (arrows in B'), leading to poorly directed movement of the cluster towards the posterior
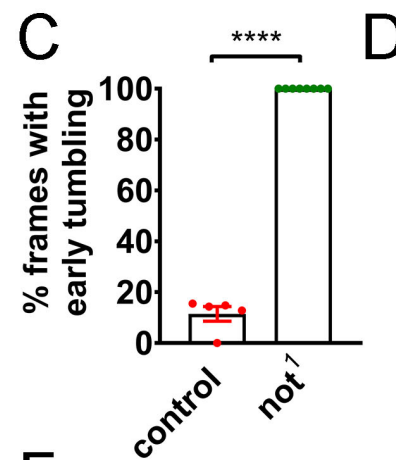

$\mathrm{F}$

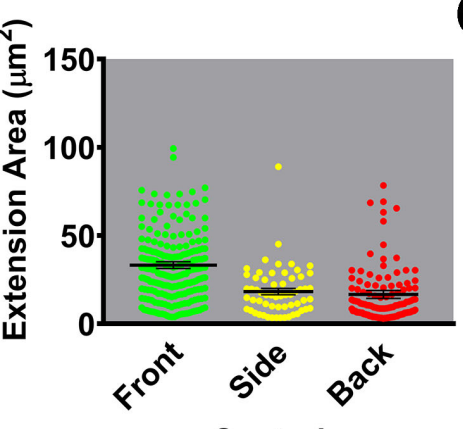

Control

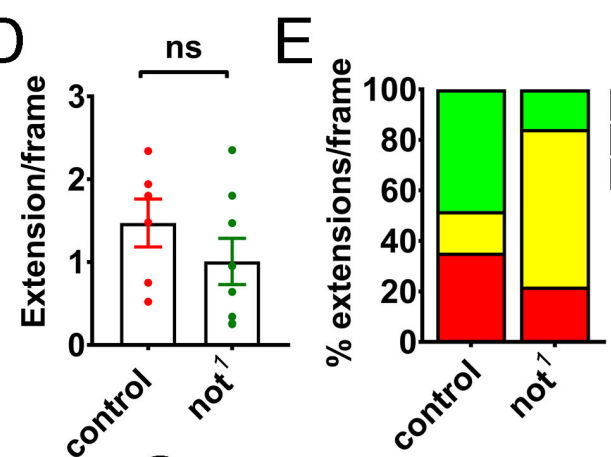

$\mathrm{G}$

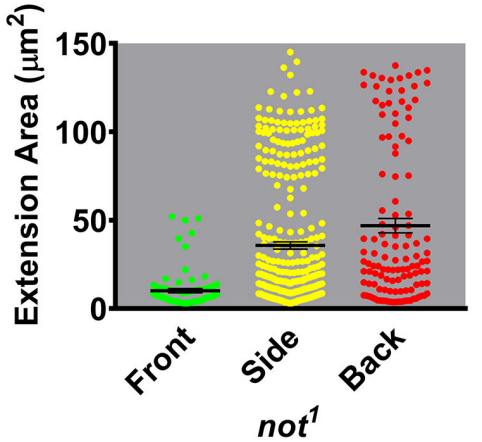
pole (track B'). C-G, Quantitation of time-lapse images from wt control $(n=5)$ and $n t^{1}(n=7)$ LifeAct-GFP-labelled

Front border cell clusters, showing effects on tumbling and Side actin-based cellular protrusions. C, Graph showing per-

Back centage of frames from the first half of migration with tumbling border cells. Individual data points are shown together with mean \pm SEM. not ${ }^{1}$ significantly increases early tumbling ***, $P<0.0001$ Student's t-test. D, Graph of total cellular extensions/frame after segmentation. There is no significant difference (ns, Student's t-test) between wt control and not ${ }^{1}$. E, Graph of percentage extensions/ frame at front, back or sides of the cluster, showing a higher proportion of extensions at the side of not $^{1}$ clusters compared to controls. F,G, Measurements of the area of extensions detected at the front, back or sides of wt and not $^{1}$ clusters, together with mean area \pm SEM, showing that the size of protrusions at the front is reduced in $n o t^{1}$ clusters concomitantly with an increase in the size of extensions at the side and back.

tumbling motion (Fig.4A-C). Further analysis revealed did not observe any difference in Scar protein staining that while there was not a global reduction in the number between not ${ }^{1}$ mutant border cells and their heterozygous of protrusions in not $^{1}$ mutant clusters (Fig.4D), there was siblings (Fig.5A,B). To test whether Scar loss-of-function a significant change in the distribution of the number phenocopied not ${ }^{1}$ clusters, we generated homozygous (Fig.4E) and size (Fig.4F,G) of protrusions, from a front clones for an amorphic Scar mutant allele, Scar ${ }^{\Delta 37}\left(\right.$ Zallen $^{\Delta}$ bias in controls (54\% of protrusions) to the sides (63\% of et al., 2002). Notably, we found F-actin polarity was unafprotrusions) in not $^{1}$ mutants (Fig.4E, $\mathrm{P}<0.01$ ), consistent fected with $\mathrm{F}$-actin being predominantly distributed at the

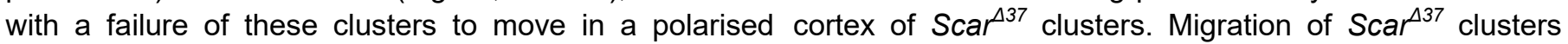
fashion.

was retarded. However, previous live imaging analysis of non-stop acts independently of Scar during border clusters in which Scar had been knocked down by RNAi, cell migration. Recent data suggest Non-stop is capable revealed that Scar loss of function resulted in a reduction of interacting with Arp2/3 and the WAVE regulatory com- in the number of cellular protrusions, with a higher proplexes (WRC) in the cytoplasm to prevent polyubiquitina- portion of protrusions at the rear of the cluster, and fewer tion and subsequent proteasomal degradation of the in the front and middle compared to controls (Law et al., WRC subunit Scar (Cloud et al., 2019). Scar/WAVE- 2013). These phenotypes are consistent with a reduction Arp2/3 interactions result in nucleation of branched actin in migration, but not with the not ${ }^{1}$ phenotypes described filament networks and in that way regulate migration above (Fig.3,4). Polarisation of the polarity determinant (Buracco et al., 2019; Krause and Gautreau, 2014). This Crb was also normal in Scar mutant clones suggesting prompted us to test whether loss of non-stop function the architecture of the clusters was unaffected. Taken resulted in destabilisation of Scar levels in border cells. together, we conclude that, in border cells, non-stop acts Endogenous Scar staining was very faint (Fig.5A,B) com- independently of Scar to drive collective migration.

pared to ectopically overexpressed Scar (Fig.5C), but we 

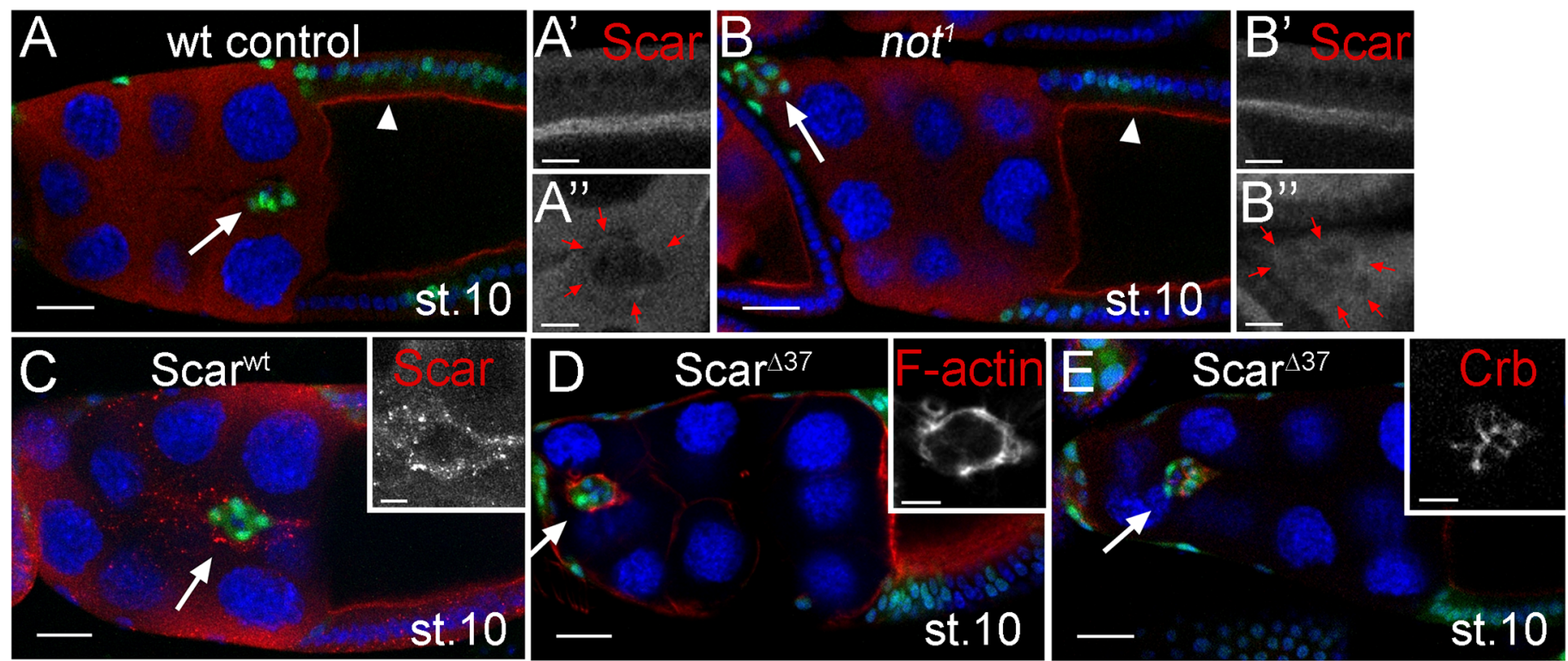

Fig.5 Non-stop acts independently of Scar during border cell migration A,B, Scar levels are not reduced in not ${ }^{1}$ follicle or border cells. Confocal micrographs showing Scar staining (red) in wt (A) and $n^{1} t^{1}$ (B) GFP-labelled MARCM clones (green) at stage 10 of egg chamber development.

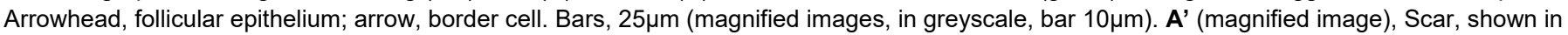
greyscale, predominantly localises to apical junctions of columnar follicle cells. A"' (magnified image), Scar staining is cytoplasmic in nurse cells but, in border cells, can be detected at the outer junctions of the cluster (red arrows). B. Scar is similarly localised in not ${ }^{1}$ clones, with no reduction in level either at the apical side of follicle cells (B') or at border cell junctions (B'). C, Overexpression of wild type Scar (Scar ${ }^{\text {wt }}$ OE) using s/boGAL4 results in a robust signal confirming of Scar staining at the outer junctions of border cells. Arrow, border cells. Bars, $25 \mu \mathrm{m}$ (inset, $10 \mu \mathrm{m}$ ). DE, Scar ${ }^{\Delta 37}$ clusters show normal F-actin and Crb polarity in migrating clusters. Shown are confocal micrographs of stage 10 egg chambers labelled with GFP (green) to mark clones of $S c a r^{\Delta 37}$ cells induced with the MARCM technique and TOPRO-3 (blue) to stain all nuclei. Bars, 25 $\mu \mathrm{m}$ (insets, $10 \mu \mathrm{m}$ ). D, Egg chamber stained with Phalloidin showing localisation of F-actin around the cortex of the cluster (arrow); E, Egg chamber stained with antibodies against $\mathrm{Crb}$, showing localisation to inner border cell junctions. Scar ${ }^{\triangle 37}$ mutant border cell clusters display defective migration, with clusters frequently failing to reach the oocyte border by stage 10 , as shown in these examples.

non-stop is required for the normal level and/or dis- around the cortex of the cluster, at the interface between tribution of Hippo signalling components in border border cells and nurse cells (Fig.6G,H). Crb is required cells. The loss of normal actin polarity, early tumbling of for polarisation of other polarity determinants, including the border cell cluster, increased polar cell number are all aPKC in border cells (Wang et al., 2018). Correspondingfeatures of Hippo signalling loss-of-function (Lin et al., ly, the distribution of aPKC was somewhat disrupted in 2014; Lucas et al., 2013). In outer border cells, the key not mutant cells (Fig.6I,J). We also observed a modest upstream components of the Hippo pathway (Crumbs, effect on the distribution of the adherens junction protein Kibra, Expanded, Merlin) are found at sites of border cell- Armadillo/ $\beta$-catenin (Arm; Fig.6K,L). Taken together, border cell contact (Lucas et al., 2013; Niewiadomska et these data show that non-stop is required for expression al., 1999), where the pathway acts independently of the of hippo signalling components and correct recruitment of canonical downstream effector Yorkie to limit the activity, polarity determinants in outer border cells.

but not the recruitment, of the actin polymerisation protein ex and mer are targets of Non-stop but not the HAT Enabled (Lucas et al., 2013). This prompted us to test module of SAGA, which is dispensable for border cell whether non-stop may be required for the normal level or migration. A key and highly conserved role of Non-stop/ distribution of Hippo signalling components in outer bor- USP22 is to regulate gene expression, acting as a central der cells. Using a transcriptional reporter of expanded component in the DUB module of the SAGA complex expression (ex-lacZ), we found a 2.46 fold reduction in (Lee et al., 2011). By exploiting genome-wide ChIPSeq expanded levels in not ${ }^{1}$ mutant cells compared to hetero- data from a recent study of the Drosophila SAGA comzygous sister cells in mosaic border cell clusters plex (Li et al., 2017), we asked whether any of the canon(Fig.6A, $\mathrm{B} ; \mathrm{P}=0.003, \mathrm{n}=26$ ). Similarly, we saw a reduction ical hippo signalling components are transcriptional tarin Merlin protein levels at border cell-border cell junctions gets of Non-stop. To do this we looked for binding sites at in not $^{1}$ mutant cells (Fig.6C,D). The distribution of Ena- the gene promoters, -1000 to $+200 \mathrm{bp}$ of the transcription bled appeared largely unaffected in $n o t^{1}$ mutant clusters start sites. We found that the expanded promoter is (Fig.6E,F). In follicle cells, Expanded and Merlin are re- bound by Non-stop ( $n=2$, Fig.7A); furthermore, depletion dundantly required for normal localisation of the apical of non-stop leads to a 2.5 fold reduction expanded extransmembrane protein Crumbs (Crb) (Aguilar-Aragon et pression in embryos ( $\mathrm{Li}$ et al., 2017), comparable to the al., 2020; Fletcher et al., 2012). Strikingly, when we ex- effect we observed in border cells (see above). Similarly, amined the distribution of $\mathrm{Crb}$, we found that rather than we also found evidence that Not binds the merlin promotbeing distributed in the junctions between neighbouring er ( $n=1$, Fig.7B). Interestingly, Ada2b, a SAGA-specific border cells (Niewiadomska et al., 1999), it was localised HAT module subunit, that anchors the HAT module to 


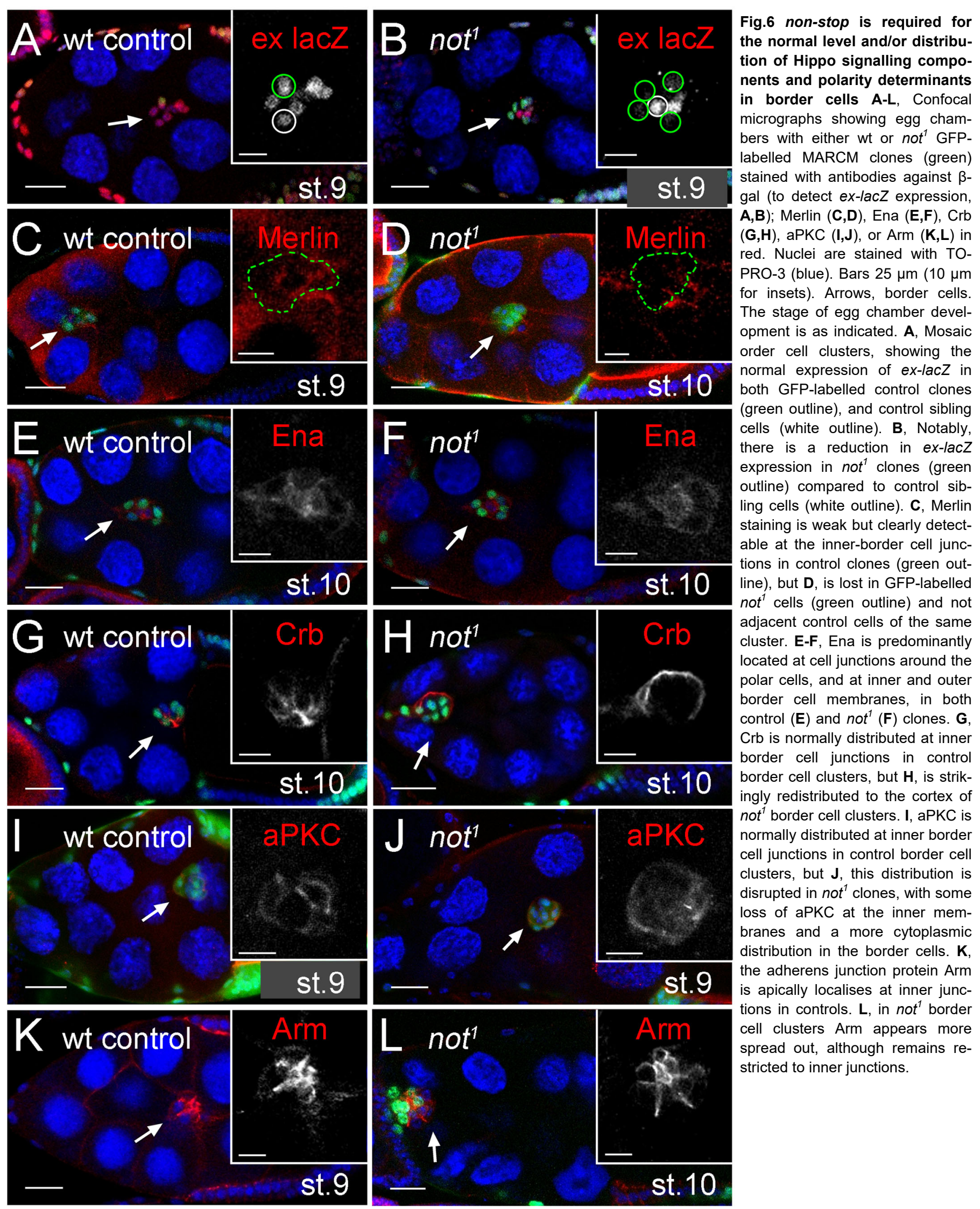

SAGA and is required for its HAT activity (Kusch et al., tested the requirement for ada $2 b$ in F-actin polarity and 2003; Lee et al., 2011; Muratoglu et al., 2003; Pankotai et border cell migration, we found that ada2b mutant border al., 2005; Zsindely et al., 2009), did not bind either of cells migrated normally with cortically-localised F-actin these loci $(n=4$, Fig.7A,B), suggesting that expanded and (Fig.7D, mean migration $82.3 \% \pm 3.6 \%, n=45$ ). Taken tomerlin promoters are DUB specific targets. Correspond- gether, these data indicate that the DUB module can regingly, we did not see a reduction in ex-lacZ levels in ulate transcription of expanded and merlin independently $a d a 2 b$ mutant clones (Fig.7C). Furthermore, when we of the HAT module in border cells. 

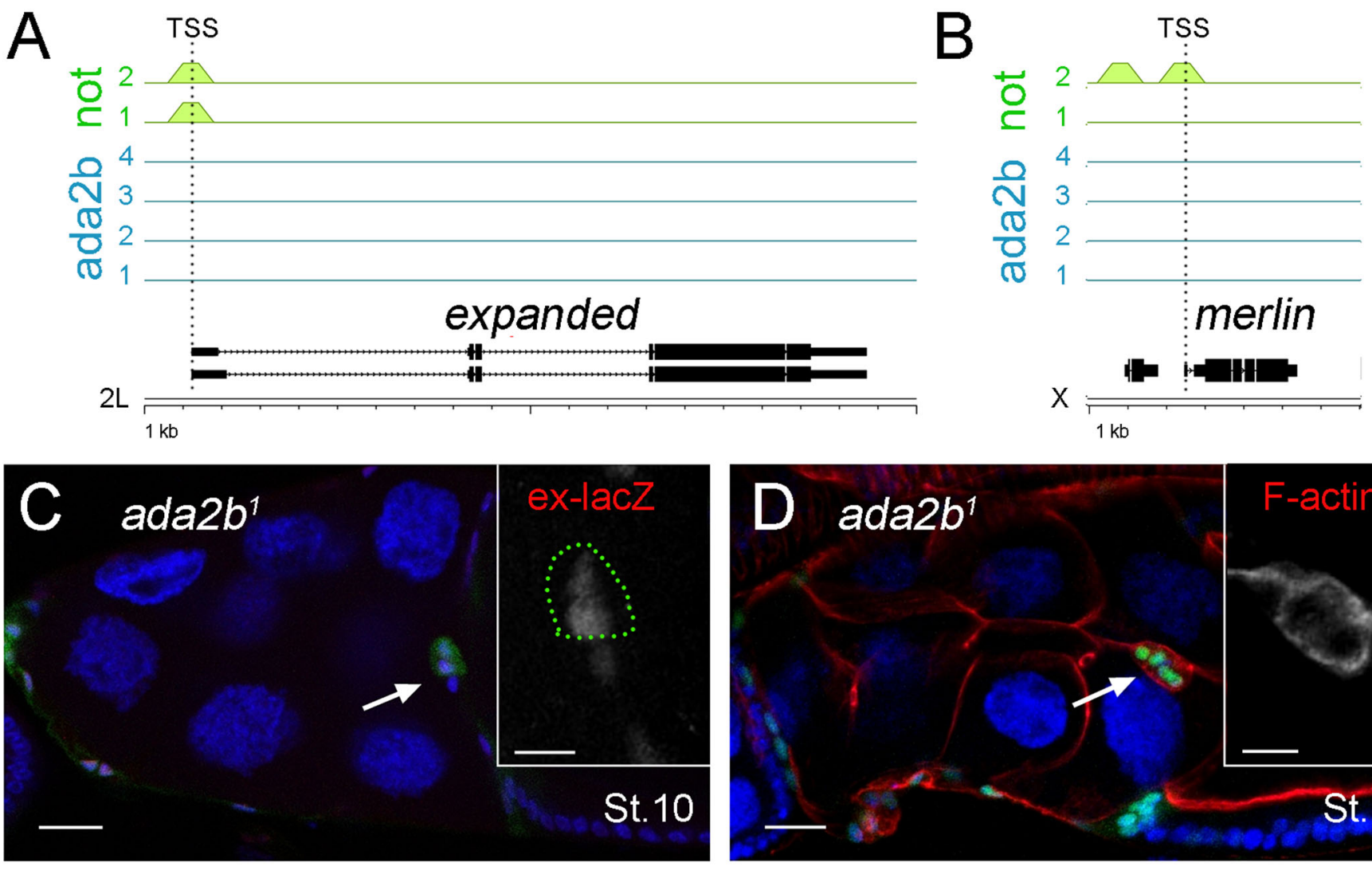

Fig.7 Ex and Mer are targets of Non-stop but not the HAT module of SAGA, which is dispensable for border cell migration. A-B, Nonstop, but not Ada2b bind to the expanded and merlin promoters. At the top are the ChIP binding profiles for all replicates of Not (green, $\mathrm{n}=2)$ and Ada2b (blue, $\mathrm{n}=4$ ) at expanded and merlin promoters in Drosophila embryos as determined from data reported in (Li et al., 2017). Position of the transcription start site (TSS) is shown with a dotted line. Below is a schematic of the gene structure at the respective genomic loci with exons (thick lines) and introns (thin lines). Scale bar, $1 \mathrm{~kb}$ intervals. C, Confocal micrograph of a stage 10 egg chamber (arrow) with ada2 ${ }^{1}$ GFP-labelled MARCM clone (green) stained with antibodies against $\beta$-gal (red) to detect ex-lacZ expression. Nuclei labelled with TO-PRO-3 (blue). Inset, exlacZ staining in grayscale, with mutant cells outlined (green dotted line). There is no reduction in ex-lacZ staining in ada $2 b^{1}$ mutant cells compared to sibling control cells. D, Confocal micrograph of a stage 10 egg chamber (arrow) with ada2 $b^{1}$ GFP-labelled MARCM clone (green) stained with Phalloidin to label F-actin (red), showing F-actin is localised to outer border cell junctions, as wild type, compare Fig2A.

Overexpression of ex partially rescues cell migration to rescue not $t^{1}$-associated defects in F-actin polarity and and polarity defects. To further explore the functional collective cell migration (Fig.8I-M). Interestingly, we also significance of reduced expanded levels, we examined saw a partial recovery in the Crb distribution in $c p b^{+} n o t^{1}$ the effect of expanded loss-of-function on border cell po- border cell clusters (Fig.8K), indicating a role for the actin larity and migration (Fig.8). We found that border cells cytoskeleton in controlling Crb polarity.

mutant for an expanded loss-of-function allele $\left(e x^{\mathrm{e} 1}\right)$ phenocopied the effect of $n o t^{1}$, albeit more weakly (Fig.8A-F), with some loss of cortical F-actin staining and a significant disruption of Crumbs distribution (Fig.8K-L), accompanied by abrogated migration (Fig.8M). Strikingly, expanded overexpression $\left(\mathrm{ex}^{+}\right)$substantially restored more normal Crumbs and F-actin distributions in not ${ }^{1}$ mutants (Fig.8G-H and K-L) and significantly suppressed the effect of not $^{1}$ on migration (Fig.8M; the mean percentage migration of $e^{+}$not $^{1}$ border cell clusters was $55.2 \pm 3.0 \%$, $\mathrm{n}=75$ compared to $38.7 \pm 2.9 \%, \mathrm{n}=101$ for not $^{1}$ alone, $\mathrm{P}<0.0001)$. Taken together with the data above, we conclude that expanded is a critical transcriptional target of non-stop required for its function in border cells. Previous studies have shown that overexpression of Capping protein $\mathrm{B}\left(c p b^{+}\right)$, which antagonises Enabled by competing for binding F-actin barbed ends and preventing actin polymerisation, is capable of complementing impaired hippo signalling (loss of warts) in border cells. Correspondingly, we find that $c p b^{+}$has a similar ability as $\mathrm{ex}^{+}$

\section{Discussion}

A non-stop-mediated transcriptional programme establishes F-actin polarity during collective migration Here we report that Drosophila USP22, encoded by nonstop, is necessary for F-actin polarity and collective cell migration of invasive border cells. Collective border cell migration requires actomyosin polymerisation and contraction at the cortex around the cluster as it moves over the nurse cell substrate; F-actin is effectively excluded from the center of the cluster where polarity determinants acting via the Hippo complex block the activity of the Factin regulator Enabled. Mechanistically, our experiments suggest non-stop regulates inside-out F-actin polarity by regulating the expression of hippo signalling components, ex and mer, which are direct Not targets. Not has been reported to regulate the actin cytoskeleton directly by promoting the stability of Scar/WAVE. However, we did not observe a reduction in Scar levels in not mutant clones and Scar loss -of-function did not disrupt F-actin polarity. 

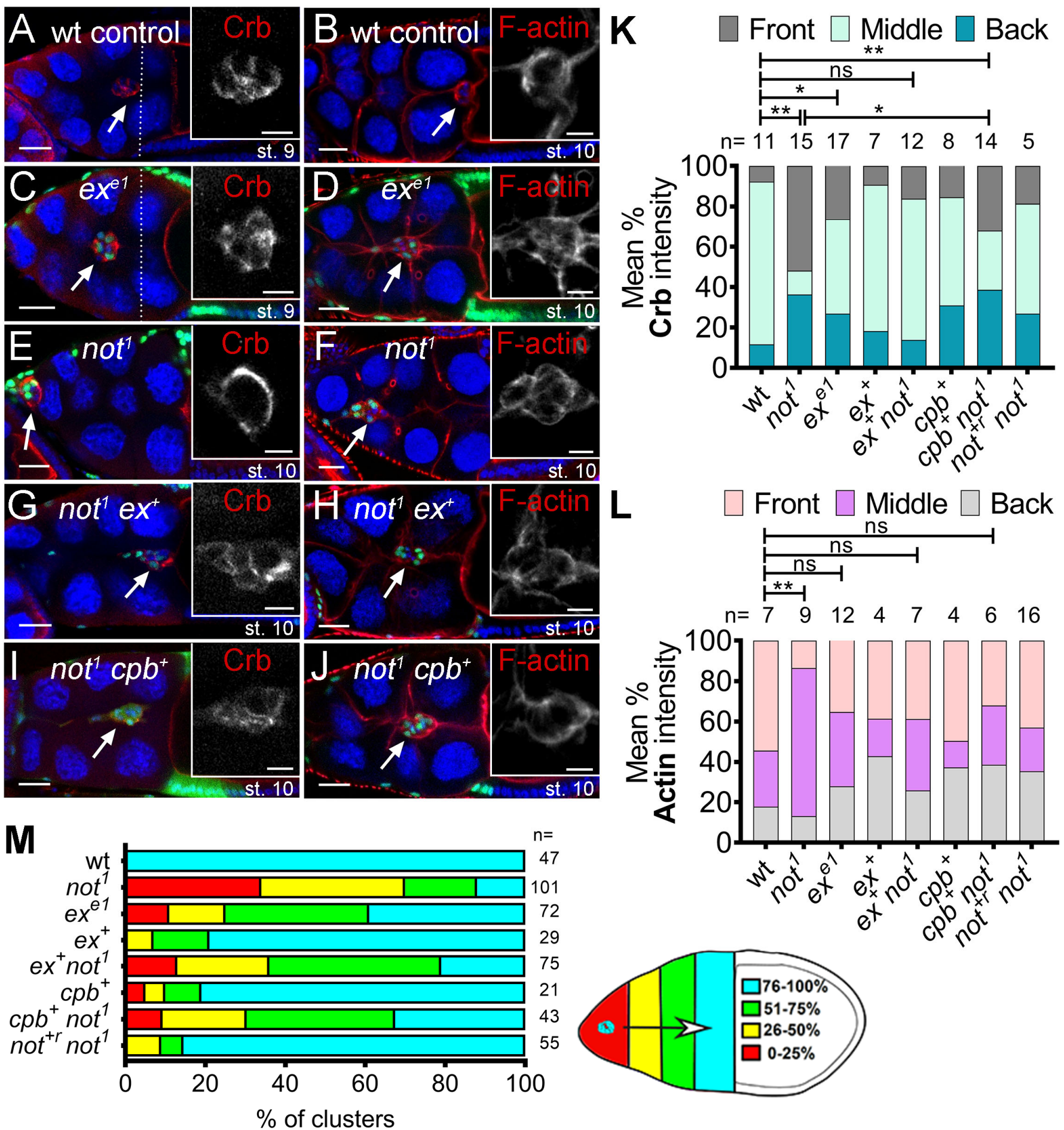

Fig.8 Overexpression of expanded or the actin capping protein $c p B$ partially rescues cell migration and polarity defects A-J, Confocal micrographs of Crb or F-actin (red) staining in egg chambers harbouring GFP-labelled MARCM clones (green) of different genotypes. TOPRO-3 (blue) labels all nuclei. Bars, $25 \mu \mathrm{m}$ (insets $10 \mu \mathrm{m}$ ). The stage of egg chamber development is as indicated, with dotted line showing position of overlying centripetal follicle cells in stage 9 chambers. Border cells are indicated with arrows. A, Control showing normal distribution of Crb at contacts between the border cells inside the cluster. B, Control showing normal cortical distribution of F-actin around the outer membrane of the cluster. C, exe ${ }^{1}$ clones showing partial disruption of Crb. D, F-actin polarisation is also partially impaired in exe ${ }^{1}$ border cells, with some F-actin visible at inner junctions of the migrating clusters. $\mathbf{E}, \mathrm{Crb}$ is redistributed away from inner junctions to the cortex of the cluster in not ${ }^{1}$ clones. F, Factin is found distributed on inner junctions of not $^{1}$ clusters between border cells. G, The disruption of Crb localisation in not ${ }^{1}$ clones is partially rescued by overexpression of ex $\left(n o t^{1} e x^{+}\right)$. $\mathbf{H}, \mathrm{F}$-actin also is more normally polarised in $n o t^{1} e x^{+}$border cells, although some weak staining is also evident between border cell-border cell junctions. I, Overexpression of $\mathrm{cpB}$ weakly restores some Crb distribution in not ${ }^{1}$ mutant cells $\left(n o t^{1} c p B^{+}\right.$) and $\mathbf{J}, \mathrm{F}$-actin is displaced from border cell junctions inside the $n t^{1} c p B^{+}$clusters. $\mathbf{K}$, Quantification of mean percentage of Crb staining at the front, middle and back of the cluster (area under curve measurements) derived from lines scans taken from several egg chambers ( $n=n u m b e r$ of clusters). ${ }^{*} \mathrm{P}<0.05$; ${ }^{* *} \mathrm{P}<0.001$; ns, not significant, 2-way Anova comparisons of mean ratio of Crb staining at the front of the cluster; comparable results were obtained for comparisons of staining in the middle of the cluster (not shown). L, Quantification of mean percentage of F-actin staining at the front, middle and back of the cluster (area under curve measurements) derived from lines scans taken from several egg chambers ( $n=$ =number of clusters). ${ }^{* *} \mathrm{P}<0.001$; ns, not significant, 2-way Anova comparisons of mean ratio of F-actin staining at the front of the cluster; comparable results were obtained for comparisons of staining in the middle of the cluster (not shown). $\mathbf{M}$, Histograms summarising border cell migration defects at stage 10 in the indicated genotypes ( $n=$ number of egg chambers), alongside the migration index for quantification of migration. 
Furthermore, we did not observe a significant change in the number of actin protrusions following not loss of function, which might be expected if Scar were a target in border cells. Notably, we find that overexpression of expanded suppressed not $^{1}$-induced $\mathrm{F}$-actin accumulation at inner border cell junctions, consistent with partial restoration of Hippo function and inhibition of Enabled function. We also observed that $c p b$ overexpression rescued loss of non -stop, again consistent with disruption of Enabled function due to competitive binding of $\mathrm{Cpb}$ to F-actin barbed ends and the inhibition of F-actin polymerisation at inner border cell junctions. Interestingly, studies of maternallyprovided not in the early embryo have identified a requirement for not in membrane invagination and nuclear anchoring during cellularisation (Li et al., 2017). Invagination is driven by actin, which is highly polarised at the base of invaginating membranes, and transiently in apical microvilli. Enabled plays an important role in establishing actin dynamics during invagination (Grevengoed et al., 2003), raising the question of whether the regulatory network between Not and the Hippo complex we have uncovered also has a role to play in this context.

non-stop regulates the distribution of polarity determinants. A striking effect of not loss of function in border cells is the redistribution of Crb from inner to outer border cell junctions. When we looked at possible effects of this on other polarity determinants, we found localisation of aPKC to the inside apical junction between border cells was disrupted, consistent with previous studies showing that Crb, acting together with the Par complex and endocytic recycling machinery, is necessary for ensuring its correct distribution (Wang et al., 2018). Mislocalised aPKC generates protrusions at the side and back of border cells (Wang et al., 2018), just as we have seen in not ${ }^{1}$ clusters. Hence, whilst loss of Hippo components leads to loss of inside-out actin polarity, disruption of $\mathrm{Crb}$ and aPKC might account for the change in orientation of protrusions. Why is Crumbs mis-localised to the cortex of the border cell complex? Our complementation experiments (Fig. 8) suggest that this might be at least partially accounted for by loss of expression of the FERM domain proteins Expanded and Merlin, which in follicle cells act together with Moesin (Moe) to recruit Crb to the apical surface (Aguilar-Aragon et al., 2020). Moe stabilises Crb at the apical membrane of epithelia by linking Crb to cortical actin (Medina et al., 2002). Although the physical interaction between Moe and Crb may be weak (Sherrard and Fehon, 2015), Moe is an important regulator of dynamic Crb localisation in follicle cells, as it acts to antagonise interactions between Crb and aPKC at the marginal zone of the apical membrane domain, while stabilising interactions between $\mathrm{Crb}$ and the apical surface (Sherrard and Fehon, 2015). Importantly, in border cells, Moe is cortically localised where it organises a supercellular actin cytoskeleton network and promotes cortical stiffness (Ramel et al., 2013). An attractive hypothesis therefore is that Moe, perhaps along with other proteins, is a sink for Crb at the cortex of the border cell cluster following loss of Ex and Mer at inner border cell junctions in non-stop mutants. When we overexpressed expanded the normal pattern of Crb localisation was partially restored, in support of a competitive binding model. Interestingly, we also observed weak rescue of Crb localisation following $\mathrm{CpB}$ overexpression. This might be because Moe, or other proteins that tether Crb on the outer membrane are only accessible in the absence of a strong supercellular F-actin cortex and that restoration of cortical F-actin in $n o t^{1} c p b^{+}$cells displaces Crb. In wild type border cells, Crb needs to be constantly moved from the outside membrane in a dynamin- and Rab5-dependent manner (Wang et al., 2018). Another possibility therefore, which is not mutually exclusive with the first, is that polarisation of the F-actin cytoskeleton is important for correct trafficking of Crb in border cells, as it is in follicle cells (Aguilar-Aragon et al., 2020).

non-stop is necessary for the expression of ex and mer independently of the requirement for $y k i$. Abnormal accumulation of F-actin in epithelial tissues, e.g. resulting from loss of $\mathrm{CpB}$, has been shown to lead to Ykiinduced expression of ex, mer, and other target genes to reinforce Hippo activity at the cortex (Fernandez et al., 2011; Ko et al., 2016; Sansores-Garcia et al., 2011). It is known that the Hippo pathway integrates multiple inputs at the level of Yki and that Yki interacts with a number of chromatin-modifying factors for transcriptional activation of target genes (Hillmer and Link, 2019). Is it possible that non-stop acts to support Yki-mediated expression of ex and mer? In border cells, ectopic overexpression of Yki has been reported to accelerate border cell migration, resulting in clusters prematurely reaching the oocyte during stage 9, suggesting that there may be a Yorkiemediated negative feedback loop to maintain F-actin homeostasis (Lucas et al., 2013). However, yki mutant border cells or clusters in which yki has been knocked down in the outer border cells migrate normally, suggesting that $y k i$ is normally dispensable in outer border cells for invasive migration (Lin et al., 2014). We therefore favour a model whereby non-stop provides independent transcriptional control of ex and mer in this context. The situation is different in polar cells, where the Hippo pathway is proposed to act by suppressing Yki activity and cell proliferation to maintain normal polar cell numbers. However, similar to Hippo loss-of-function or yki gain-of-function, we find that loss of non-stop leads to increased numbers of polar cells, which, again, argues against a role for nonstop in supporting $y k i$-mediated gene expression. Nevertheless, what this does suggest is that the requirement for non-stop in Hippo complex formation is not limited to situations where the Hippo complex acts in a $y k i-$ independent fashion.

SAGA-independent roles for non-stop during development and disease. The growth, specification and migration of cells during tissue development requires precisely regulated patterns of gene expression, that depend on numerous cues for temporal and spatial gene activa- 
tion, involving crosstalk with multiple signalling pathways. Strikingly, it has emerged that factors once considered to be ubiquitous regulators of transcription, including the SAGA chromatin-modifying complex, can have specific roles in discrete developmental processes. Although it has been suggested that SAGA is required for all transcribed genes in some contexts (Bonnet et al., 2014), numerous studies have shown that loss of SAGA components affects the expression of only a subset of genes (Pahi et al., 2015; Pankotai et al., 2013; Zsindely et al., 2009) and different components modulate distinct and overlapping subsets (Helmlinger et al., 2008; Helmlinger et al., 2011; Lee et al., 2000; Weake et al., 2008). These differences in expression are likely to explain their different physiological roles; for instance, during female germline development in Drosophila, ada2B affects the expression of many genes and is required for oogenesis, whereas non-stop affects relatively few and is dispensable (Li et al., 2017). Elegant genome-wide ChIP studies indicate that even though both DUB and HAT modules bind the same genes, many of the targets do not require the DUB module for expression, explaining the observed dependencies. These experiments also reveal nonoverlapping sites of chromatin occupancy for the DUB and HAT modules of SAGA in Drosophila (Li et al., 2017), but the significance of differences in transcriptional targeting for cell function had not been established. Notably, in this respect, we find that the requirement for non-stop in border cell migration is not matched by a requirement for $a d a 2 b$. Furthermore, Ada2b has not been found to bind the ex and mer promoters, providing a molecular explanation for non-stop's SAGA-independent role. Importantly, these findings challenge the perceived view that transcriptional roles for non-stop/USP22 are mediated solely by SAGA. This may have broader relevance to situations where USP22, but not other members of SAGA are associated with human disease states, particularly where cell polarity is frequently disrupted, such as cancer (Glinsky et al., 2005). Our current efforts are directed at identifying SAGA-independent factors that facilitate Nonstop's chromatin binding and function.

\section{Methods}

Non-stop transgene. An RNAi-resistant, full-length nonstop expression construct was synthesised by GeneArt (Invitrogen). RNAi-resistance was achieved by incorporating numerous silent polymorphic mutations, such that, in the regions targeted by dsRNAs, homology with the inverted repeat sequences was limited to no more than 8 contiguous base pairs (Jonchere and Bennett, 2013). The non-stop open reading frame was shuttled into pPMWattB (Chen et al., 2015) by gateway cloning, placing the non-stop open-reading frame downstream of a Myc epitope tag. Stable transgenic flies were made by phiC31 integrase-mediated transgenesis at a landing site on the second (attP40, at 25C6) and third (attP2, at 68A4) chromosomes by the Cambridge fly facility (University of Cambridge).
Drosophila stocks and genetics. Flies were raised and crossed at $25^{\circ} \mathrm{C}$ according to standard procedures. $w^{1118}$ or FRT80B flies were used as the wild-type control strains. 138 RNAi lines, corresponding to 45 Drosophila DUBs (details available on request), were screened for border cell defects at $25^{\circ} \mathrm{C}$. UAS-not ${ }^{\prime R}$ (Vienna Drosophila Resource Center \#45776) was identified as having the most severe effect on migration. The FLP/FRT site-specific recombination system was used to generate mutant clones with a heat-shock promoter (Xu and Rubin, 1993). The following fly lines were obtained from the Bloomington Drosophila Stock Center: FRT80B (BL1988), $w^{1118}$ (BL6409), slbo-Gal4, UAS-GFP (BL6458, Montell Lab), slbo-lacZ enhancer trap line (BL12227), slbo-Lifeact-GFP (BL58364), c306-Gal4, UAS-GFP (BL3743). For clonal analysis we used the following strains:

hsFLP, tub-Gal4, UAS-GFP; +/+; tubGAL80 FRT80B/ TM6B (generated from BL42732, BL5191),

hsFLP, tub-Gal4, UAS-GFP; tubGAL80 FRT40A/+; +/ TM6B (generated from BL42732, BL5192),

hsFLP, tub-Gal4, UAS-GFP; +/+; FRT82B tubGAL80/

TM6B (generated from BL42732, BL44408).

The amorphic not allele, not ${ }^{1}$ was obtained from Margarete Heck and recombined with FRT80B. FRT82B Ada2B was a gift from Jerry Workman (Li et al., 2017). UAS-Scar and FRT40A Scar ${ }^{\triangle 37}$ were gifts from Eyal Schejter. UAS-cpB, UAS-ex (Lucas et al., 2013), updlacZ (Jiang et al., 2009) and ex-lacZ (Fletcher et al., 2012), were gifts from Nic Tapon. Information on these strains is also available at http://www.flybase.org.

Generation of mosaic clones using MARCM. Mosaic Analysis with a Repressible Cell Marker (MARCM) was used to generate positively marked clones labelled with GFP (Lee and Luo, 2001). Expression of genes under GAL4-UAS is inhibited in the presence of GAL80. Heat shocking induces the expression of heat shock (hs) driven FLP, which acts to induce recombination at Flippase Recognition Targets (FRT). Homozygous daughter cells lacking GAL80 are then capable of GAL4-mediated gene expression of GFP and other UAS-transgenes. Mitotic recombination is initiated after heat shock where some daughter cells are $\mathrm{GFP}^{+}$while others are GFP- due to the presence of GAL80. To obtain border cell mitotic (mosaic) clones, progeny of the right genotypes were heat shocked twice a day for 1 hour each with at least 5 $\mathrm{hr}$ intervals between treatments, from pupae to adult at $37^{\circ} \mathrm{C}$. Newly enclosed adults (2-3 d old) were fattened for $2 \mathrm{~d}$ on yeast paste.

Immunofluorescent staining. Ovaries were dissected in PBS (Phosphate buffer saline) and fixed with 3.7\% paraformaldehyde in PBS. The ovaries were washed with PBST (1x PBS, 0.2\% Tween 20) 3 times for 15 minutes each time. Ovaries were then blocked with PBTB (1x PBS, $0.2 \%$ Tween $20,5 \%$ fetal bovine serum) for 1 hour at room temperature. The ovaries were treated with primary antibodies in PBTB at $4^{\circ} \mathrm{C}$ overnight. The following 
primary antibodies were used for immunostaining. Developmental Studies Hybridoma Bank (DSHB): mouse antiArmadillo (N27A1, 1:200, concentrate), mouse antiEnabled (5G2, 1:25, concentrate), mouse anti- $\beta$-gal (401a, 1:300, concentrate), mouse anti-eyes absent (eya10H6, 1:100, supernatant), mouse anti-SCAR (P1C1, $1: 200$, concentrate). Mouse anti-aPKC $\zeta$ (sc-17781, 1:200) from Santa Cruz. Guinea pig anti-Merlin (1:7500) from $R$ Fehon lab. The primary antibodies were washed with PBST 3 times $15 \mathrm{~min}$ and then blocked with PBTB for $1 \mathrm{hr}$ at room temperature. Ovaries were incubated with Alexafluor-conjugated secondary antibodies (1:500, Life technologies) in PBTB at $4^{\circ} \mathrm{C}$ overnight. Phalloidin 555 (1:50, Molecular Probes) was used to stain F-actin. Ovaries were washed with PBST for 15 minutes before staining nuclei with TO-PRO-3 (Life technologies, 1:1000) in PBST for 15 minutes. Ovaries were mounted in Vectashield (Vector laboratories). For Crumbs staining, Ovaries were dissected in PBS (Phosphate buffer saline) and fixed with boiled $8 \%$ paraformaldehyde in PBS and heptane $(6: 1)$ for 10 minutes. Samples were treated with heptane and methanol (1:2) for 30 seconds. They were then washed in methanol for 10 minutes. The ovaries were washed with PBST (1x PBS, 0.2\% Tween 20) 2 times for 15 minutes each time. Ovaries were then blocked with PBTB (1x PBS, 0.2\% Tween 20, 5\% fetal bovine serum) for 30 minutes at room temperature. The ovaries were treated with mouse anti-Crumbs (Cq4, 1:100, concentrate, DSHB) in PBTB at $4^{\circ} \mathrm{C}$ overnight.

Image acquisition and analysis of fixed samples. Images were taken on a confocal microscope (LSM710 or LSM780, Carl Zeiss) using 20x/0.5NA air objectives. Three laser lines were used based on the excitation of wavelength of the staining dyes which includes $488 \mathrm{~nm}$, $561 \mathrm{~nm}$ and $633 \mathrm{~nm}$ wavelengths. Extent of migration (the migration index) was measured as a percentage of the distance travelled to the oocyte/nurse cell boundary in stage 10 egg chambers. ImageJ (https://imagej.nih.gov/ ij/) was used for quantification of signal intensities in mosaic clusters using z-stack maximum projections. Raw integrated density was used as intensity values. For line scan profiles, maximum intensity images of Actin and Crumbs staining were generated in ImageJ. Background signal were subtracted. The plot profile function in ImageJ was used to measure signal intensities along lines drawn through the centre of border cell clusters and the peak analyser tool in OriginPro (Origin Lab) was used to calculate the area under peaks that were identified. The ratio of intensities at front, middle and back, were compared and normalised in Prism8 (Graphpad). The following statistical tests were performed using Prism 8 (GraphPad): Student's t-tests; one-way or two-way Anova, with Tukey correction for multiple comparisons; multiple linear regression with least squares. Figures were made using FigureApp in OMERO (Allan et al., 2012; Burel et al., 2015) and final assembly in Adobe Photoshop.
Egg chamber culture and time-lapse imaging of live egg chambers. Live imaging of egg chamber culture were as previously described (Law et al., 2013; Prasad et al., 2007) with slight modification. Briefly, media for both dissection and live-imaging, comprised of Schneider media (Gibco), 15\% fetal bovine serum, $0.1 \mathrm{mg} / \mathrm{ml}$ acidified insulin (Sigma), 9 MM FM4-64 dye (Molecular Probes) and $0.1 \mathrm{mg} / \mathrm{ml}$ Pen-strep (Gibco) was freshly prepared. The $\mathrm{pH}$ of the media was adjusted to 6.90-6.95. Individual egg chambers from well fattened progeny of the right genotype were dissected and transferred to borosilicate glass bottom chambered coverglasses (ThermoFisher) for imaging. Imaging was done at $25^{\circ} \mathrm{C}$. Time-lapse movies were acquired on an inverted confocal microscope (LSM 710; Carl Zeiss) using 20x/0.5NA air objectives. Two laser lines were used based on the excitation of wavelength of the endogenous GFP and FM4-64 dye, which are $488 \mathrm{~nm}$, and $561 \mathrm{~nm}$ wavelengths respectively. $16-20$ slices of Z-stacks were taken with $2.5 \mu \mathrm{m}$ slices every $3 \mathrm{~min}$.

Analysis of time-lapse images. Time-lapse image analyses were performed using a custom macro for ImageJ to analyse the behaviour of border cell migration and extension dynamics (Law et al., 2013; Poukkula et al., 2011) with slight modification. Briefly, time-lapse movies were split into different channels. Maximum projections of the GFP-channel were created. Egg chambers were rotated so that anterior ends were at the left. Border cells were manually thresholded to mask nuclear GFP generated from the MARCM system through the first or early phase of migration. Images of border cells clusters were then segmented into cell body and cellular extensions using signals from slbo-LifeAct-GFP. Extensions were grouped based on their positions in relation to the leading edge of the cluster: front $\left(315-45^{\circ}\right)$, side $\left(45-135^{0}\right.$ or $\left.225-315^{\circ}\right)$ and back $\left(135-225^{\circ}\right)$. The macro also enabled tracking of the movement of cluster to measure the migration speed. Forward directed speed was calculated on $\mathrm{x}$-axis by taking distance of the centre of cluster at one time point relative to the next time point. The tumbling index was calculated as the mean percentage of frames per time lapse movie that showed rounded clusters, exhibiting changes in the position of individual cells within the cluster for two or more consecutive frames in the first half of migration. Data were collated in Microsoft Excel and independent Student's t-tests were done with Prism 8 (GraphPad). For visualisation of stills (Fig4A,A'-B,B'), GFP-labelled nuclei were segmented in Imaris (Bitplane) and labelled in white.

Analysis of previously reported ChIP datasets. ChIPseq data were downloaded from GEO (https:// www.ncbi.nlm.nih.gov/geo/) using accession GSE98862; the dm3 assembly of the $D$. melanogaster genome was obtained from UCSC (http://www.genome.ucsc.edu/cgibin/hgTables). Peaks from Ada2b and Non-stop ChIP experiments were mapped to the dm3 genome assembly using BEDtools software (Quinlan and Hall, 2010), and 
any genes matching to peaks from -1000 to +200 of the Fig. 5

transcription start site (TSS) were identified. For visuali- A, A' and A". hsFLP, tub-Gal4, UAS-GFP/+ ;; +, FRT80B/ sation of ChIP-seq peaks on the genome, we utilised the tub-Gal80, FRT80B

'karyoploteR' R/Bioconductor package (Gel and Serra, B, B' and B". hsFLP, tub-Gal4, UAS-GFP/+ ;; not', 2017).

FRT80B/tub-Gal80, FRT80B

\section{Genotypes of strains}

Fig 1.

A. $w^{1118} /+$; SIbo-Gal4, UAS-GFP/+

B. SIbo-Gal4, UAS-GFP/UAS-not ${ }^{1 R}$

C. c306-Gal4, UAS-GFP; UAS-not ${ }^{+r} /+$

E. (as A-C with) Slbo-Gal4, UAS-GFP/UAS-not $t^{R}$; UAS$\underline{n o t^{+r} / t}$

F. hsFLP, tub-Gal4, UAS-GFP/+ ;; +, FRT80B/tub-Gal80, FRT80B

G,I. hsFLP, tub-Gal4, UAS-GFP/+ ;; not ${ }^{1}$, FRT80B/tubGal80, FRT80B

$\mathrm{H}$. (as $F, G$ with) hsFLP, tub-Gal4, UAS-GFP/+ ; UASnot $^{+r} /+$; not $^{1}$, FRT80B/tub-Gal80, FRT80B

Fig. 2

A. hsFLP, tub-Gal4, UAS-GFP/+; slbo-lacZ/+; +, FRT80B/tub-Gal80, FRT80B

B. hsFLP, tub-Gal4, UAS-GFP/+; slbo-lacZ/+; not ${ }^{1}$, FRT80B/tub-Gal80, FRT80B

C. Quantification of $A, B$ :

Wild type GFP: $h s F L P$, tub-Gal4, UAS-GFP/+; slbo-lacZ/ +; +, FRT80B/tub-Gal80, FRT80B (or homozygous for tub-Gal80, FRT80B)

Wild type GFP ${ }^{+}$: hsFLP, tub-Gal4, UAS-GFP/+; slbo-lacZ/ +; +, FRT80B/+, FRT80B

not ${ }^{1}$ GFP: : hsFLP, tub-Gal4, UAS-GFP/+; slbo-lacZ/+; not ${ }^{1}$, FRT80B/tub-Gal80, FRT80B (or homozygous for tub-Gal80, FRT80B)

not ${ }^{1}$ GFP $^{+}:$hsFLP, tub-Gal4, UAS-GFP/+; slbo-lacZ/+; not $^{1}$, FRT80B/ not $^{1}$, FRT80B

D. hsFLP, tub-Gal4, UAS-GFP/+ ;; +, FRT80B/tub-Gal80, FRT80B

E. hsFLP, tub-Gal4, UAS-GFP/+ ; not ${ }^{1}$, FRT80B/tubGal80, FRT80B

F. hsFLP, tub-Gal4, UAS-GFP/upd-lacZ ;; +, FRT80B/tub -Gal80, FRT80B

G,H. hsFLP, tub-Gal4, UAS-GFP/upd-lacZ ;; not ${ }^{1}$, FRT80B/tub-Gal80, FRT80B

$\mathrm{I}, \mathrm{J}$. (quantification of $\mathrm{F}-\mathrm{H}$ )

Fig. 3

wt control: hsFLP, tub-Gal4, UAS-GFP/+ ;; +, FRT80B/ tub-Gal80, FRT80B

not $^{1}$ : hsFLP, tub-Gal4, UAS-GFP/+ ;; not ${ }^{1}$, FRT80B/tubGal80, FRT80B

not $^{1}$; tub>not ${ }^{+r}$ : hsFLP, tub-Gal4, UAS-GFP/+ ; UAS-not ${ }^{+r} /$

+ ; not ${ }^{1}$, FRT80B/tub-Gal80, FRT80B

Fig. 4

Control: hsFLP, tub-Gal4, UAS-GFP/+; slbo-LifeAct-GFP/ +; +, FRT80B/tub-Gal80, FRT80B

not $^{\prime}$ : hsFLP, tub-Gal4, UAS-GFP/+; slbo-LifeAct-GFP/+; not $^{1}$, FRT80B/tub-Gal80, FRT80B
C. Slbo-Gal4, UAS-GFP/UAS-Scar ${ }^{\text {wt }}$

D-E. hsFLP; tubGAL80, FRT40A/ Scar ${ }^{437}$, FRT40A; Act $>C D 2>$ Gal4, UAS-GFP/+

Fig. 6.

A (wt control): hsFLP, tub-Gal4, UAS-GFP/+ ; ex-lacZ/+; ,+ FRT80B/tub-Gal80, FRT80B

B $\left(\right.$ not $\left.^{1}\right)$ : hsFLP, tub-Gal4, UAS-GFP/+ ; ex-lacZ/+; not ${ }^{1}$, FRT80B/tub-Gal80, FRT80B

C, E, G, I, K (wt control): hsFLP, tub-Gal4, UAS-GFP/+ ;; + , FRT80B/tub-Gal80, FRT80B

$\mathrm{D}, \mathrm{F}, \mathrm{H}, \mathrm{J}, \mathrm{L}\left(\right.$ not $\left.^{1}\right)$ : hsFLP, tub-Gal4, UAS-GFP/+ ;; not ${ }^{1}$, FRT80B/tub-Gal80, FRT80B

Fig. 7

C. hsFLP, tub-Gal4, UAS-GFP/+ ; ex-lacZ/+; ada2b ${ }^{1}$, FRT82B/tub-Gal80, FRT82B

D. hsFLP, tub-Gal4, UAS-GFP/+ ;; ada $2 b^{1}$, FRT82B/tubGal80, FRT82B

Fig. 8

A, B. hsFLP, tub-Gal4, UAS-GFP/+; ; +, FRT80B/tubGal80, FRT80B

C, D. hsFLP; ex ${ }^{e 1}, \quad F R T 40 A / t u b-G a l 80, \quad F R T 40 A$; Act $>C D 2>G a / 4, U A S-G F P /+$

E, F. hsFLP, tub-Gal4, UAS-GFP/+; ; not ${ }^{1}$, FRT80B/tubGal80, FRT80B

G, H. hsFLP, tub-Gal4, UAS-GFP/+; UAS-ex ${ }^{+} /+;$not $^{1}$, FRT80B/tub-Gal80, FRT80B

I, J. hsFLP, tub-Gal4, UAS-GFP/+; UAS-cpB ${ }^{+} /+;$not $^{1}$, FRT80B/tub-Gal80, FRT80B

$\mathrm{K}-\mathrm{M}$, (quantitation of A-J together with the following genotypes)

hsFLP, tub-Gal4, UAS-GFP/+; UAS-ex+/+; +, FRT80B/tub -Gal80, FRT80B

hsFLP, tub-Gal4, UAS-GFP/+; UAS-cpB ${ }^{+} /+;$+, FRT80B/ tub-Gal80, FRT80B

hsFLP, tub-Gal4, UAS-GFP/+ ; UAS-not ${ }^{+r} /+$; not $^{1}$, FRT80B/tub-Gal80, FRT80B

\section{Online supplementary material}

Video S1: $4 \mathrm{~h}$ time-lapse of border cell migration starting from specification of the cluster and the ability of the cluster to acquire forward protrusion, followed by cell-on-cell migration to the anterior border of the oocyte. GFP expression is driven by s/bo-Gal4 to label the border cell cluster in green. Nuclei are labelled with Ub-His2A-RFP in magenta.

Video S2: $4 \mathrm{~h}$ time-lapse movie of normal border cell migration showing onset of migration including the ability of cluster to acquire forward actin protrusions. MARCM clones are labelled with nuclear GFP, F-actin is labelled with LifeAct-GFP. Egg chamber genotype: hsFLP, tub- 
Gal4, UAS-GFP/+; slbo-LifeAct-GFP/+; +, FRT80B/tubGal80, FRT80B.

Video S3: $4 \mathrm{~h}$ time-lapse movie of abnormal border cell migration showing early tumbling of the cluster and multidirectional actin protrusions in $n t^{1}$ mutant cells labelled with nuclear GFP using MARCM. F-actin is labelled with LifeAct-GFP. Egg chamber genotype: hsFLP, tub-Gal4, UAS-GFP/+; slbo-LifeAct-GFP/+; not ${ }^{1}, \quad$ FRT80B/tubGal80, FRT80B

\section{Acknowledgments}

We thank Rick Fehon, Margarete Heck, Timothy Megraw, Eyal Schejter, Nic Tapon, Jerry Workman the Developmental Studies Hybridoma Bank (DSHB), and Bloomington Stock Center for antibodies, vectors and fly stocks. Thanks also to the Liverpool Computational Biology Facility and Chris Seidel (Stowers Institute) for assistance with ChIP data analysis, and to the Liverpool Centre for Cell Imaging (https://cci.liv.ac.uk/) for help with microscopy and image analysis. The work was funded by the MRC (MR/K015931/1), NWCR (CR847), Liverpool CRUK Centre and the University of Liverpool international $\mathrm{PhD}$ fees waiver scheme.

\section{References}

Abdelilah-Seyfried, S., D.N. Cox, and Y.N. Jan. 2003. Bazooka is a permissive factor for the invasive behavior of discs large tumor cells in Drosophila ovarian follicular epithelia. Development. 130:1927-1935.

Aguilar-Aragon, M., G. Fletcher, and B.J. Thompson. 2020. The cytoskeletal motor proteins Dynein and MyoV direct apical transport of Crumbs. Dev Biol. 459:126-137.

Allan, C., J.M. Burel, J. Moore, C. Blackburn, M. Linkert, S. Loynton, D. Macdonald, W.J. Moore, C. Neves, A. Patterson, M. Porter, A. Tarkowska, B. Loranger, J. Avondo, I. Lagerstedt, L. Lianas, S. Leo, K. Hands, R.T. Hay, A. Patwardhan, C. Best, G.J. Kleywegt, G. Zanetti, and J.R. Swedlow. 2012. OMERO: flexible, model-driven data management for experimental biology. Nat Methods. 9:245-253.

Bai, J., and D. Montell. 2002. Eyes absent, a key repressor of polar cell fate during Drosophila oogenesis. Development. 129:5377-5388.

Bai, J., Y. Uehara, and D.J. Montell. 2000. Regulation of invasive cell behavior by taiman, a Drosophila protein related to AIB1, a steroid receptor coactivator amplified in breast cancer. Cell. 103:1047 $-1058$.

Beccari, S., L. Teixeira, and P. Rorth. 2002. The JAK/STAT pathway is required for border cell migration during Drosophila oogenesis. Mech Dev. 111:115 -123 .
Bianco, A., M. Poukkula, A. Cliffe, J. Mathieu, C.M. Luque, T.A. Fulga, and P. Rorth. 2007. Two distinct modes of guidance signalling during collective migration of border cells. Nature. 448:362-365.

Bonnet, J., C.Y. Wang, T. Baptista, S.D. Vincent, W.C. Hsiao, M. Stierle, C.F. Kao, L. Tora, and D. Devys. 2014. The SAGA coactivator complex acts on the whole transcribed genome and is required for RNA polymerase II transcription. Genes Dev. 28:1999-2012.

Buracco, S., S. Claydon, and R. Insall. 2019. Control of actin dynamics during cell motility. F1000Res. 8.

Burel, J.M., S. Besson, C. Blackburn, M. Carroll, R.K. Ferguson, H. Flynn, K. Gillen, R. Leigh, S. Li, D. Lindner, M. Linkert, W.J. Moore, B. Ramalingam, E. Rozbicki, A. Tarkowska, P. Walczysko, C. Allan, J. Moore, and J.R. Swedlow. 2015. Publishing and sharing multi-dimensional image data with OMERO. Mamm Genome. 26:441-447.

Cai, D., S.C. Chen, M. Prasad, L. He, X. Wang, V. Choesmel-Cadamuro, J.K. Sawyer, G. Danuser, and D.J. Montell. 2014. Mechanical feedback through E-cadherin promotes direction sensing during collective cell migration. Cell. 157:11461159.

Cai, J., M.K. Culley, Y. Zhao, and J. Zhao. 2018. The role of ubiquitination and deubiquitination in the regulation of cell junctions. Protein Cell. 9:754-769.

Chen, J.V., L.R. Kao, S.C. Jana, E. Sivan-Loukianova, S. Mendonca, O.A. Cabrera, P. Singh, C. Cabernard, D.F. Eberl, M. Bettencourt-Dias, and T.L. Megraw. 2015. Rootletin organizes the ciliary rootlet to achieve neuron sensory function in Drosophila. J Cell Biol. 211:435-453.

Clague, M.J., I. Barsukov, J.M. Coulson, H. Liu, D.J. Rigden, and S. Urbe. 2013. Deubiquitylases from genes to organism. Physiol Rev. 93:1289-1315.

Cloud, V., A. Thapa, P. Morales-Sosa, T.M. Miller, S.A. Miller, D. Holsapple, P.M. Gerhart, E. Momtahan, J.L. Jack, E. Leiva, S.R. Rapp, L.G. Shelton, R.A. Pierce, S. Martin-Brown, L. Florens, M.P. Washburn, and R.D. Mohan. 2019. Ataxin-7 and Nonstop coordinate SCAR protein levels, subcellular localization, and actin cytoskeleton organization. Elife. 8.

Duchek, P., and P. Rorth. 2001. Guidance of cell migration by EGF receptor signaling during Drosophila oogenesis. Science. 291:131-133.

Duchek, P., K. Somogyi, G. Jekely, S. Beccari, and P. Rorth. 2001. Guidance of cell migration by the Drosophila PDGF/VEGF receptor. Cell. 107:17-26.

Fernandez, B.G., P. Gaspar, C. Bras-Pereira, B. Jezowska, S.R. Rebelo, and F. Janody. 2011. Actin-Capping Protein and the Hippo pathway regulate F-actin and tissue growth in Drosophila. Development. 138:2337-2346. 
Fletcher, G.C., E.P. Lucas, R. Brain, A. Tournier, and B.J. Thompson. 2012. Positive feedback and mutual antagonism combine to polarize Crumbs in the Drosophila follicle cell epithelium. Curr Biol. 22:1116-1122.

Fulga, T.A., and P. Rorth. 2002. Invasive cell migration is initiated by guided growth of long cellular extensions. Nat Cell Biol. 4:715-719.

Gel, B., and E. Serra. 2017. karyoploteR: an R/ Bioconductor package to plot customizable genomes displaying arbitrary data. Bioinformatics. 33:3088-3090.

Glinsky, G.V., O. Berezovska, and A.B. Glinskii. 2005. Microarray analysis identifies a death-from-cancer signature predicting therapy failure in patients with multiple types of cancer. J Clin Invest. 115:1503-1521.

Godt, D., and U. Tepass. 2009. Breaking a temporal barrier: signalling crosstalk regulates the initiation of border cell migration. Nat Cell Biol. 11:536-538.

Grevengoed, E.E., D.T. Fox, J. Gates, and M. Peifer. 2003. Balancing different types of actin polymerization at distinct sites: roles for Abelson kinase and Enabled. J Cell Biol. 163:1267-1279.

Haeger, A., K. Wolf, M.M. Zegers, and P. Friedl. 2015. Collective cell migration: guidance principles and hierarchies. Trends Cell Biol. 25:556-566.

Helmlinger, D., S. Marguerat, J. Villen, S.P. Gygi, J. Bahler, and F. Winston. 2008. The S. pombe SAGA complex controls the switch from proliferation to sexual differentiation through the opposing roles of its subunits Gen5 and Spt8. Genes Dev.

22:3184-3195.

Helmlinger, D., S. Marguerat, J. Villen, D.L. Swaney, S.P. Gygi, J. Bahler, and F. Winston. 2011. Tra1 has specific regulatory roles, rather than global functions, within the SAGA co-activator complex. EMBO J. 30:2843-2852.

Hillmer, R.E., and B.A. Link. 2019. The Roles of Hippo Signaling Transducers Yap and Taz in Chromatin Remodeling. Cells. 8.

Jang, A.C., Y.C. Chang, J. Bai, and D. Montell. 2009. Border-cell migration requires integration of spatial and temporal signals by the BTB protein Abrupt. Nat Cell Biol. 11:569-579.

Jiang, H., P.H. Patel, A. Kohlmaier, M.O. Grenley, D.G. McEwen, and B.A. Edgar. 2009. Cytokine/Jak/ Stat signaling mediates regeneration and homeostasis in the Drosophila midgut. Cell. 137:13431355.

Jonchere, V., and D. Bennett. 2013. Validating RNAi phenotypes in Drosophila using a synthetic RNAiresistant transgene. PLoS One. 8:e70489.

Ko, C., Y.G. Kim, T.P. Le, and K.W. Choi. 2016. Twinstar/ cofilin is required for regulation of epithelial in- tegrity and tissue growth in Drosophila. Oncogene. 35:5144-5154.

Kosinsky, R.L., F. Wegwitz, N. Hellbach, M. Dobbelstein, A. Mansouri, T. Vogel, Y. Begus-Nahrmann, and S.A. Johnsen. 2015. Usp22 deficiency impairs intestinal epithelial lineage specification in vivo. Oncotarget. 6:37906-37918.

Koutelou, E., C.L. Hirsch, and S.Y. Dent. 2010. Multiple faces of the SAGA complex. Curr Opin Cell Biol. 22:374-382.

Krause, M., and A. Gautreau. 2014. Steering cell migration: lamellipodium dynamics and the regulation of directional persistence. Nat Rev Mol Cell Biol. 15:577-590.

Kusch, T., S. Guelman, S.M. Abmayr, and J.L. Workman. 2003. Two Drosophila Ada2 homologues function in different multiprotein complexes. Mol Cell Biol. 23:3305-3319.

Law, A.L., A. Vehlow, M. Kotini, L. Dodgson, D. Soong, E. Theveneau, C. Bodo, E. Taylor, C. Navarro, U. Perera, M. Michael, G.A. Dunn, D. Bennett, R. Mayor, and M. Krause. 2013. Lamellipodin and the Scar/WAVE complex cooperate to promote cell migration in vivo. J Cell Biol. 203:673-689.

Lee, K.K., M.E. Sardiu, S.K. Swanson, J.M. Gilmore, M. Torok, P.A. Grant, L. Florens, J.L. Workman, and M.P. Washburn. 2011. Combinatorial depletion analysis to assemble the network architecture of the SAGA and ADA chromatin remodeling complexes. Mol Syst Biol. 7:503.

Lee, T., and L. Luo. 2001. Mosaic analysis with a repressible cell marker (MARCM) for Drosophila neural development. Trends Neurosci. 24:251-254.

Lee, T.I., H.C. Causton, F.C. Holstege, W.C. Shen, N. Hannett, E.G. Jennings, F. Winston, M.R. Green, and R.A. Young. 2000. Redundant roles for the TFIID and SAGA complexes in global transcription. $\mathrm{Na}$ ture. 405:701-704.

Li, X., C.W. Seidel, L.T. Szerszen, J.J. Lange, J.L. Workman, and S.M. Abmayr. 2017. Enzymatic modules of the SAGA chromatin-modifying complex play distinct roles in Drosophila gene expression and development. Genes Dev. 31:1588-1600.

Lin, T.H., T.H. Yeh, T.W. Wang, and J.Y. Yu. 2014. The Hippo pathway controls border cell migration through distinct mechanisms in outer border cells and polar cells of the Drosophila ovary. Genetics. 198:1087-1099.

Lin, Z., H. Yang, Q. Kong, J. Li, S.M. Lee, B. Gao, H. Dong, J. Wei, J. Song, D.D. Zhang, and D. Fang. 2012. USP22 antagonizes $p 53$ transcriptional activation by deubiquitinating Sirt1 to suppress cell apoptosis and is required for mouse embryonic development. Mol Cell. 46:484-494.

Lucas, E.P., I. Khanal, P. Gaspar, G.C. Fletcher, C. Polesello, N. Tapon, and B.J. Thompson. 2013. 
The Hippo pathway polarizes the actin cytoskeleton during collective migration of Drosophila border cells. J Cell Biol. 201:875-885.

Margolis, J., and A. Spradling. 1995. Identification and behavior of epithelial stem cells in the Drosophila ovary. Development. 121:3797-3807.

Martin, K.A., B. Poeck, H. Roth, A.J. Ebens, L.C. Ballard, and S.L. Zipursky. 1995. Mutations disrupting neuronal connectivity in the Drosophila visual system. Neuron. 14:229-240.

McDonald, J.A., A. Khodyakova, G. Aranjuez, C. Dudley, and D.J. Montell. 2008. PAR-1 kinase regulates epithelial detachment and directional protrusion of migrating border cells. Curr Biol. 18:16591667.

McDonald, J.A., E.M. Pinheiro, and D.J. Montell. 2003. PVF1, a PDGF/VEGF homolog, is sufficient to guide border cells and interacts genetically with Taiman. Development. 130:3469-3478.

Medina, E., J. Williams, E. Klipfell, D. Zarnescu, G. Thomas, and A. Le Bivic. 2002. Crumbs interacts with moesin and beta(Heavy)-spectrin in the apical membrane skeleton of Drosophila. J Cell Biol. 158:941-951.

Mishra, A.K., J.P. Campanale, J.A. Mondo, and D.J. Montell. 2019. Cell interactions in collective cell migration. Development. 146.

Montell, D.J., P. Rorth, and A.C. Spradling. 1992. slow border cells, a locus required for a developmentally regulated cell migration during oogenesis, encodes Drosophila C/EBP. Cell. 71:51-62.

Montell, D.J., W.H. Yoon, and M. Starz-Gaiano. 2012. Group choreography: mechanisms orchestrating the collective movement of border cells. Nat Rev Mol Cell Biol. 13:631-645.

Muratoglu, S., S. Georgieva, G. Papai, E. Scheer, I. Enunlu, O. Komonyi, I. Cserpan, L. Lebedeva, E. Nabirochkina, A. Udvardy, L. Tora, and I. Boros. 2003. Two different Drosophila ADA2 homologues are present in distinct GCN5 histone acetyltransferase-containing complexes. Mol Cell Biol. 23:306321.

Niewiadomska, P., D. Godt, and U. Tepass. 1999. DECadherin is required for intercellular motility during Drosophila oogenesis. J Cell Biol. 144:533547.

Norden, C., and V. Lecaudey. 2019. Collective cell migration: general themes and new paradigms. Curr Opin Genet Dev. 57:54-60.

Pahi, Z., Z. Kiss, O. Komonyi, B.N. Borsos, L. Tora, I.M. Boros, and T. Pankotai. 2015. dTAF10- and dTAF10b-Containing Complexes Are Required for Ecdysone-Driven Larval-Pupal Morphogenesis in Drosophila melanogaster. PLoS One. 10:e0142226.
Pankotai, T., O. Komonyi, L. Bodai, Z. Ujfaludi, S. Muratoglu, A. Ciurciu, L. Tora, J. Szabad, and I. Boros. 2005. The homologous Drosophila transcriptional adaptors ADA2a and ADA2b are both required for normal development but have different functions. Mol Cell Biol. 25:8215-8227.

Pankotai, T., N. Zsindely, E.E. Vamos, O. Komonyi, L. Bodai, and I.M. Boros. 2013. Functional characterization and gene expression profiling of Drosophila melanogaster short dADA2b isoformcontaining dSAGA complexes. BMC Genomics. $14: 44$

Pinheiro, E.M., and D.J. Montell. 2004. Requirement for Par-6 and Bazooka in Drosophila border cell migration. Development. 131:5243-5251.

Plutoni, C., S. Keil, C. Zeledon, L.E.A. Delsin, B. Decelle, P.P. Roux, S. Carreno, and G. Emery. 2019. Misshapen coordinates protrusion restriction and actomyosin contractility during collective cell migration. Nat Commun. 10:3940.

Poeck, B., S. Fischer, D. Gunning, S.L. Zipursky, and I. Salecker. 2001. Glial cells mediate target layer selection of retinal axons in the developing visual system of Drosophila. Neuron. 29:99-113.

Poukkula, M., A. Cliffe, R. Changede, and P. Rorth. 2011. Cell behaviors regulated by guidance cues in collective migration of border cells. J Cell Biol. 192:513-524.

Prasad, M., A.C. Jang, M. Starz-Gaiano, M. Melani, and D.J. Montell. 2007. A protocol for culturing Drosophila melanogaster stage 9 egg chambers for live imaging. Nat Protoc. 2:2467-2473.

Quinlan, A.R., and I.M. Hall. 2010. BEDTools: a flexible suite of utilities for comparing genomic features. Bioinformatics. 26:841-842.

Ramel, D., X. Wang, C. Laflamme, D.J. Montell, and G. Emery. 2013. Rab11 regulates cell-cell communication during collective cell movements. Nat Cell Biol. 15:317-324.

Sansores-Garcia, L., W. Bossuyt, K. Wada, S. Yonemura, C. Tao, H. Sasaki, and G. Halder. 2011. Modulating $\mathrm{F}$-actin organization induces organ growth by affecting the Hippo pathway. EMBO J. 30:2325-2335.

Schumacher, L. 2019. Collective Cell Migration in Development. Adv Exp Med Biol. 1146:105-116.

Sherrard, K.M., and R.G. Fehon. 2015. The transmembrane protein Crumbs displays complex dynamics during follicular morphogenesis and is regulated competitively by Moesin and aPKC. Development. 142:1869-1878.

Silver, D.L., and D.J. Montell. 2001. Paracrine signaling through the JAK/STAT pathway activates invasive behavior of ovarian epithelial cells in Drosophila. Cell. 107:831-841. 
Stuelten, C.H., C.A. Parent, and D.J. Montell. 2018. Cell motility in cancer invasion and metastasis: insights from simple model organisms. Nat Rev Cancer. 18:296-312.

Swatek, K.N., and D. Komander. 2016. Ubiquitin modifications. Cell Res. 26:399-422.

Wang, H., Z. Qiu, Z. Xu, S.J. Chen, J. Luo, X. Wang, and J. Chen. 2018. aPKC is a key polarity determinant in coordinating the function of three distinct cell polarities during collective migration. Development. 145.

Weake, V.M., J.O. Dyer, C. Seidel, A. Box, S.K. Swanson, A. Peak, L. Florens, M.P. Washburn, S.M. Abmayr, and J.L. Workman. 2011. Posttranscription initiation function of the ubiquitous SAGA complex in tissue-specific gene activation. Genes Dev. 25:1499-1509.

Weake, V.M., K.K. Lee, S. Guelman, C.H. Lin, C. Seidel, S.M. Abmayr, and J.L. Workman. 2008. SAGAmediated $\mathrm{H} 2 \mathrm{~B}$ deubiquitination controls the development of neuronal connectivity in the Drosophila visual system. EMBO J. 27:394-405.

Weake, V.M., and J.L. Workman. 2008. Histone ubiquitination: triggering gene activity. Mol Cell. 29:653663.

Xu, T., and G.M. Rubin. 1993. Analysis of genetic mosaics in developing and adult Drosophila tissues. Development. 117:1223-1237.

Zallen, J.A., Y. Cohen, A.M. Hudson, L. Cooley, E. Wieschaus, and E.D. Schejter. 2002. SCAR is a primary regulator of Arp2/3-dependent morphological events in Drosophila. J Cell Biol. 156:689701.

Zhang, X.Y., M. Varthi, S.M. Sykes, C. Phillips, C. Warzecha, W. Zhu, A. Wyce, A.W. Thorne, S.L. Berger, and S.B. McMahon. 2008. The putative cancer stem cell marker USP22 is a subunit of the human SAGA complex required for activated transcription and cell-cycle progression. $\mathrm{Mol}$ Cell. 29:102-111.

Zsindely, N., T. Pankotai, Z. Ujfaludi, D. Lakatos, O. Komonyi, L. Bodai, L. Tora, and I.M. Boros. 2009. The loss of histone $\mathrm{H} 3$ lysine 9 acetylation due to dSAGA-specific $d A d a 2 b$ mutation influences the expression of only a small subset of genes. $\mathrm{Nu}$ cleic Acids Res. 37:6665-6680. 